University of Vermont

UVM ScholarWorks

2020

\title{
A Software Defined Radio Interrogator for Passive Harmonic Transponders
}

Timothy Fennell Laracy

University of Vermont

Follow this and additional works at: https://scholarworks.uvm.edu/graddis

Part of the Engineering Commons

\section{Recommended Citation}

Laracy, Timothy Fennell, "A Software Defined Radio Interrogator for Passive Harmonic Transponders" (2020). Graduate College Dissertations and Theses. 1306.

https://scholarworks.uvm.edu/graddis/1306

This Thesis is brought to you for free and open access by the Dissertations and Theses at UVM ScholarWorks. It has been accepted for inclusion in Graduate College Dissertations and Theses by an authorized administrator of UVM ScholarWorks. For more information, please contact scholarworks@uvm.edu. 


\title{
A Software Defined Radio INTERROGATOR FOR PASSIVE HARMONIC TRANSPONDERS
}

\author{
A Thesis Presented \\ by \\ Timothy F. Laracy \\ to \\ The Faculty of the Graduate College \\ of \\ The University of Vermont \\ In Partial Fulfillment of the Requirements \\ for the Degree of Master of Science \\ Specializing in Electrical Engineering
}

October, 2020

Defense Date: August 27th, 2020

Thesis Examination Committee:

Jeff Frolik, Ph.D., Advisor

Adrian Del Maestro, Ph.D., Chairperson

Tian Xia, Ph.D.

Cynthia J. Forehand, Ph.D., Dean of Graduate College 


\section{Abstract}

Passive wireless sensors provide an opportunity for long term monitoring of remote environments. Since these devices are not battery powered, they can be deployed for an indefinite amount of time. Such devices are energized by an interrogation signal that enables them to transmit information via a return signal. Some systems (e.g., RFID) utilize the same frequency for interrogation and the return, which causes unwanted interference, particularly in cluttered environments. This work considers an interrogation system for a different class of passive devices, i.e., passive harmonic transponders. Specifically, results are presented for a single-board software defined radio (SDR) interrogation system which transmits an interrogation signal at $1.3 \mathrm{GHz}$ and receives a return at $2.6 \mathrm{GHz}$. The system is demonstrated with a passive, chip-less device known as a frequency doubling reflectenna (FDR). The SDR platform enables a compact, low-cost, and quickly operating design. The mean absolute error of the proposed interrogator was found to be $1.15 \mathrm{~dB}$ when compared with laboratory-grade instrumentation. Additionally, this system is capable of interrogating up to a distance of $70 \mathrm{~cm}$ with an EIRP of only $0 \mathrm{dBm}$.

This thesis presents an SDR system made with the open-source software package, GNURadio, capable of interrogating harmonic transponders with a single, full-duplex board. All signal processing is conducted on a laptop computer, eliminating the need for expensive laboratory instrumentation. The size of the system interrogator was also minimized, reducing the form factor of the whole interrogator to be only $25 \times$ $15 \mathrm{~cm}$. Furthermore, a new harmonic transponder was designed using transmission line matching methods, eliminating the need for discrete matching components. This harmonic transponder has a conversion loss of $31.04 \mathrm{~dB}$ at an input power of -28.21 $\mathrm{dBm}$ as demonstrated with the SDR interrogator. 
To my family 


\section{ACKNOWLEDGEMENTS}

First, I would like to thank my family for supporting me through the first 5 years of my engineering career. Mom, Dad, and Aidan, I couldn't have done it without you all.

I would also like to give a big thanks to my lab mates and friends, Evan Fennelly and Blake Hewgill. Long nights in the lab studying for exams and making deadlines was made much easier with these two brilliant, driven people. I am positive they will both make excellent engineers in the years to come.

Dr. Tian Xia and Dr. Adrian Del Maestro, I would like to thank you both for serving on my committee. The classes I took with both of you inspired me and made me think.

Finally, I would like to thank my advisor, Dr. Jeff Frolik. Dr. Frolik opened my eyes to a whole aspect of electrical engineering a few years ago when I took signals and systems and communications systems. In my graduate research he has been a great source of knowledge and a insightful mentor.

This work was funded by the Vermont Space Grant Consortium under NASA Cooperative Agreement NNX15AP86H. 


\section{TABle of Contents}

Dedication ............................. ii

Acknowledgements . . . . . . . . . . . . . . iii

List of Figures . . . . . . . . . . . . . . . . . . . . vii

List of Tables . . . . . . . . . . . . . . . . . viii

1 Introduction $\quad 1$

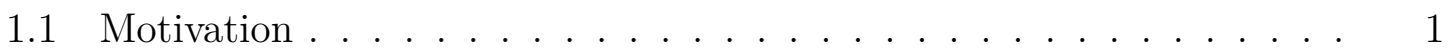

1.2 Problem Statement . . . . . . . . . . . . . . . . . . . 4

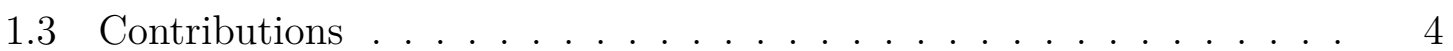

1.4 Thesis Layout . . . . . . . . . . . . . . . . . . 5

2 Background $\quad 7$

2.1 Passive Transponder Technologies . . . . . . . . . . . . . . . . . . 7

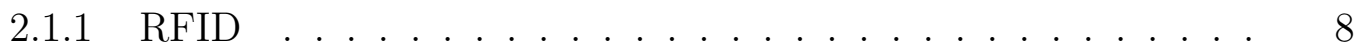

2.1 .2 SAW . . . . . . . . . . . . . . . . . 9

2.1.3 Harmonic Transponders . . . . . . . . . . . . . . . . . . . . 10

2.2 Harmonic Radar Systems . . . . . . . . . . . . . . . . . . . . . 11

2.2.1 Small Creature Tracking . . . . . . . . . . . . . . . . 12

2.2.2 Sensing and Harsh Environments . . . . . . . . . . . . . . . . 13

2.3 Frequency Doubling Reflectenna . . . . . . . . . . . . . . . . . . 14

2.4 An Introduction to Software Defined Radio . . . . . . . . . . . . . . . 15

2.4.1 Typical Hardware in SDR . . . . . . . . . . . . . . . . 17

2.4 .2 The Nuand BladeRF SDR . . . . . . . . . . . . . . . . . 18

2.4 .3 GNU Radio . . . . . . . . . . . . . . . . . . . . . 19

2.5 SDR for Spectral Analysis . . . . . . . . . . . . . . . . . 20

2.6 Conclusion . . . . . . . . . . . . . . . . . . . . . . 22

3 Harmonic Transponder Design 23

3.1 Patch Antennas . . . . . . . . . . . . . . . . . . . . . . . 23

3.1 .1 Design . . . . . . . . . . . . . . . . . 24

3.1.2 Final Patch Antennas . . . . . . . . . . . . . . 25

3.2 Diode Doubler. . . . . . . . . . . . . . . . . . . 26

3.2 .1 Doubler Impedance . . . . . . . . . . . . . . . . . 27

3.2.2 Matching ..................... . . . 29

3.3 Simulation Results . . . . . . . . . . . . . . . . . . . . 32

3.3.1 Antennas with Matching Networks . . . . . . . . . . . 33

3.3.2 Conversion Loss . . . . . . . . . . . . . . . . . . . . . . . . . . 34

3.4 Final Harmonic Transponder Layout . . . . . . . . . . . . . . . 35 
3.5 Conclusion . . . . . . . . . . . . . . . . . . 37

4 Software Defined Radio Interrogator $\quad 38$

4.1 Forward . . . . . . . . . . . . . . . . . . . . . . . . . . . . . . . . . .

4.2 Interrogator Software . . . . . . . . . . . . . . . . . . . . . . . . . . . . . . .

4.3 Results . . . . . . . . . . . . . . . . . . . . . 42

4.4 Conclusion . . . . . . . . . . . . . . . . . . . 45

5 Miniturizing Interrogator and Testing with Harmonic Transonder 46

5.1 Introduction . . . . . . . . . . . . . . . . 46

5.2 Filter and Amplifier Board Design . . . . . . . . . . . . . . 47

5.2.1 Amplifier Layout . . . . . . . . . . . . . . . . . . . . 48

5.2 .2 SMT Splitter . . . . . . . . . . . . . . . . . . . 49

5.2 .3 Filtering . . . . . . . . . . . . . . . . . . . . 49

5.2 .4 Final PCB Layout . . . . . . . . . . . . . . . 50

5.3 Measurement Results . . . . . . . . . . . . . . . . . . . 50

5.3 .1 Return Loss . . . . . . . . . . . . . . . . . 51

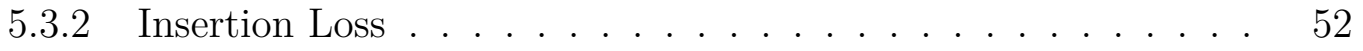

5.4 Interrogation . . . . . . . . . . . . . . . . 53

5.4.1 Calculated Transponder Metrics . . . . . . . . . . . . 55

5.4.2 Conclusion . . . . . . . . . . . . . . . . . . 57

6 Conclusions and Future Work 59

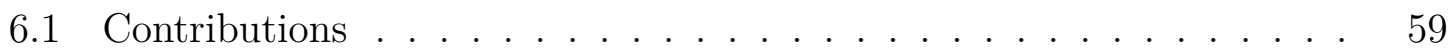

6.2 Future Work - Interdigital Capacitor . . . . . . . . . . . . . 61

6.3 Final Words . . . . . . . . . . . . . . . . . 63

$\begin{array}{ll}\text { A Interrogator Code (Python) } & 68\end{array}$ 


\section{List OF FiguRES}

1.1 A complete harmonic transponder system . . . . . . . . . . . . . . . 2

2.1 A general RFID tag. The microchip is responsible for modulating backscattered information with digital data encoded in the chip. Image from $[1]$. . . . . . . . . . . . . . . . . . . . . . . . .

2.2 Diagram of a typical SAW tag. These devices modulate electromagnetic energy using an acoustic medium. . . . . . . . . . . . . . . 9

2.3 General harmonic transponder layout . . . . . . . . . . . . . . . . . . 10

2.4 Example of loop harmonic transponder from [2]. Loop diameter is 1 mm. . . . . . . . . . . . . . . . . . 12

2.5 Layout of frequency doubling reflectenna (FDR) from [3] . . . . . . 16

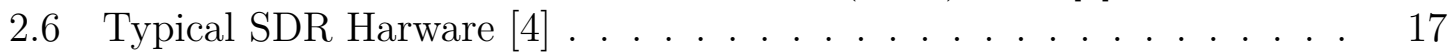

2.7 Nuand Bladerfx40 SDR . . . . . . . . . . . . . . . . . . . . 19

2.8 Example of a GNU Radio flow graph . . . . . . . . . . . . . . . . . . 20

2.9 (L) Time domain. Continuous wave (CW) tone with and without window applied. (R) Frequency domain. DFT of CW tone with and without window applied. . . . . . . . . . . . . . . . 22

3.1 Dimensions of a typical patch antenna . . . . . . . . . . . . . . 24

3.2 Final antennas designs. $1.3 \mathrm{GHz}$ antenna $(75.5 \mathrm{~mm} \times 60 \mathrm{~mm})$ is on the left, and $2.6 \mathrm{GHz}$ antenna $(37.7 \mathrm{~mm} \times 30 \mathrm{~mm})$ is on the right. . . 26

3.3 Diode model for Skyworks SMS7630 with SC-79 package. Capacitor and inductor are parasitics of the SC-79 package. . . . . . . . . . . 27

3.4 Input $(1.293 \mathrm{GHz})$ and output $(2.586 \mathrm{GHz})$ diode doubler impedances 28

3.5 Diagram of single stub matching network . . . . . . . . . . . . . . 29

3.6 Return loss of $1.3 \mathrm{GHz}$ antenna with matching network, relative to diode doubler input impedance. Resonant frequency ranges from 1.29 to $1.31 \mathrm{GHz}$. . . . . . . . . . . . . . . . . . .

3.7 Return loss of $2.6 \mathrm{GHz}$ antenna with matching network, relative to diode doubler output impedance. Resonant frequency is found to be $2.619 \mathrm{GHz}$

3.8 Conversion loss of diode doubler with matching circuits connected . . 35

3.9 Final harmonic transponder design . . . . . . . . . . . . . 36

4.1 Diagram of filtering scheme used for SDR-interrogator . . . . . . . . . 39

4.2 Program flowchart of the SDR-based interrogator. "TX" and "RX" are the separate transmit and receive chains of the BladeRF. . . . . . 40 
4.3 SDR interrogator setup. Distance from antenna to FDR is $70 \mathrm{~cm}$. Anechoic foam is used around the FDR during testing to minimize scattering effects. . . . . . . . . . . . . . . .

4.4 SDR vs. signal generator and spectrum analyzer testing of FDR. Transmit EIRP set to $8 \mathrm{dBm}$. Interrogation distance is $70 \mathrm{~cm}$. Peak return frequency is $2.565 \mathrm{GHz}$ (FDR is resonant at $1.2825 / 2.5650 \mathrm{GHz}$ ). 42

4.5 Swept transmitted power with associated receive power. Antenna placed $70 \mathrm{~cm}$ away from FDR. . . . . . . . . . . . . . . . 43

4.6 TX power set to $0 \mathrm{dBm}$. 5 averages is sufficient for the FDR peak frequency to be $3 \mathrm{~dB}$ above the noise floor. . . . . . . . . . . . . . . 44

5.1 Filter and amplifier flow chart . . . . . . . . . . . . . . . 47

5.2 Amplifier layout for Minicircuits GALI-29+ . . . . . . . . . . . 48

5.3 Filter and amplifier board layout . . . . . . . . . . . . . 51

5.4 S11 of both RX and TX Path (ports on left side of Figure 5.3 . . . . 52

5.5 S21 response of the both RX and TX path . . . . . . . . . . 53

5.6 Final setup for interrogating harmonic transponder . . . . . . . . . 54

5.7 Harmonic transponder at a distance of $53.3 \mathrm{~cm}$ from the antenna. $\mathrm{SG}+\mathrm{SA}$ setup is the same as used in Chapter 4. The EIRP of the setup is $5 \mathrm{dBm} \ldots \ldots \ldots \ldots . \ldots \ldots 5$

6.1 Simulated capacitance varied from $0.5-2 \mathrm{pF}$. Resultant shift in peak is $23 \mathrm{MHz}$. . . . . . . . . . . . . . . . 62

$6.22 .6 \mathrm{GHz}$ antenna layout with interdigital capacitor integrated. . . . . 62 


\section{LIST OF TABLES}

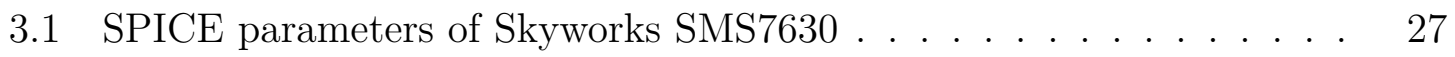

5.1 Link budget for calculating the conversion loss of the harmonic transpon-

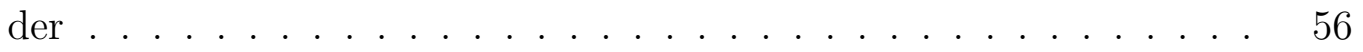




\section{Chapter 1}

\section{INTRODUCTION}

\subsection{Motivation}

The use of embedded sensing has increased significantly in recent years with the growth of the Internet of Things and the desire for flexible, robust sensing systems. Often times the sensing device (or node) will operate at very low power, allowing it to stay where it was deployed for long periods of time without maintenance. For some applications it may be desired for the sensor to have no battery at all, allowing the device to have a very long life. These devices are called passive devices . Passive wireless sensing technologies, such as micro-electro-mechanical systems (MEMS), surface acoustic wave (SAW), and radio frequency identification (RFID) offer a distinct advantage over typical wireless sensors, as they do not need batteries, but can still be interrogated remotely.

Wireless sensing is of particular importance to NASA's Integrated Vehicle Health Management systems (IVHM) $[5,6]$. IVHM is critical in space travel for keeping the crew safe and the spacecraft in good working condition. Seeing as several applications 


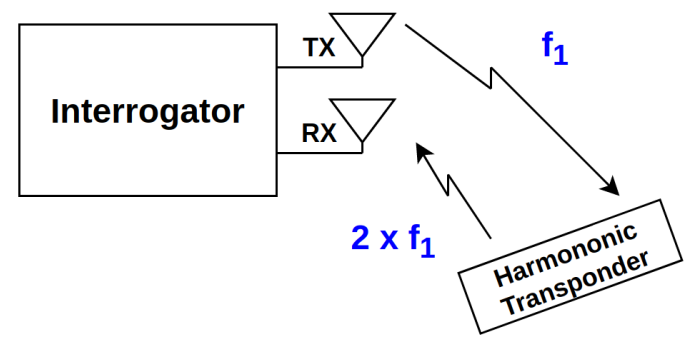

Figure 1.1: A complete harmonic transponder system

for spacecraft flight or testing have to operate in harsh environments (sometimes temperatures greater than $1500{ }^{\circ} \mathrm{C}[7]$ ), wireless sensors that are battery powered (i.e., active) are not an option. Wired sensors are also not ideal, since wiring can be prone to breaks and degradation in such environments. Therefore, the need for wireless, rugged, low-cost passive sensors is of high importance. Current passive technologies such as SAW, RFID, and MEMS have found their place in many different projects for NASA $[8,9]$. These types of devices are also used in our everyday lives, such as in ski passes and automatic toll collection systems [10,11].

A technology not currently leveraged by NASA is known as harmonic radar. Harmonic transponders are tags used to double the incedent radar signal, and send it back to the harmonic radar. A harmonic transponders consists of two antennas, with a radio frequency diode in the middle (i.e., a very simple, passive device). An interrogator sends a signal of frequency $f_{1}$ to the device. The device sends the signal through the RF diode which induces harmonics in the received signal. The signal is then radiated out of the transmit antenna tuned to operate at twice the frequency that came into the receive antenna. By measuring the second harmonic of the signal, interference between the interrogation $\left(f_{1}\right)$ and the receive $\left(2 \times f_{1}\right)$ can be eliminated, making harmonic radar especially useful for cluttered environments. The work in [12] 
used harmonic radar to measure corrosion in large industrial tanks by placing a chemically reactive element in the harmonic radar tag. Similarly, work from [13] used a dielectric resonator antenna (DRA), where the dielectric has a known temperature coefficient. A shift in the resonance peak in the device, denoted a reliable change in temperature.

The University of Vermont has researched various characteristics of wireless sensor networks over the past several years. Notable, is work done with a device called the frequency doubling reflectenna (FDR), which is a specific model of harmonic transponder [14]. The work in [14] used the FDR to measure soil characteristics for structural health monitoring (SHM) applications. Even with no embedded sensing, soil moisture could still be measured solely with the strength of the received signal. Other work shows that minimum activation power of a FDR is about $-30 \mathrm{dBm}$, considerably lower than activation power for RFID, being around -14 dBm [3].

Often times, interrogators for harmonic radar systems can be quite large, and bulky $[13,14]$. A technology called software defined radio (SDR) offers a compact, unique solution to interrogating harmonic transponders. SDR's are radio's that are highly configurable, since all base-band processing is done is software. These radios can easily become receivers for any known modulation scheme, such as FM radio band or IEEE 802.15.4. SDR's that are full-duplex allow the radio to both transmit and receive at the same time. Often times, the transmit and receive ports can also be tuned to separate frequencies, which can be done in an SDR called the Nuand BladeRF [15], making SDR perfect for interrogating harmonic transponders. 


\subsection{Problem Statement}

This thesis strives to answer the following question: can we leverage low cost hardware and manufacturing processes to design an accurate harmonic transponder interrogator? Harmonic transponder interrogators are often quite expensive and bulky [16-18]. With the main goal of this work being to miniaturize the interrogation system, we focus on making the interrogator as small and accurate as possible while still using low cost components. Consumer SDR's have a very large price range, but boards can often be bought for under $\$ 500$. Furthermore, with the rise of maker's and electronic hobbyists, PCB manufacturing costs a fraction of what it did earlier. This allows us to maintain a low cost using these services and other low cost components.

\subsection{Contributions}

In this thesis, three main contributions are made.

1. Develop software capable of measuring received signals from harmonic transponder This task was done using the commercial off-the-shelf (COTS) SDR called the Nuand BladeRF [15]. The BladeRF is an open source SDR platform capable of tuning anywhere between $300 \mathrm{MHz}$ and $3.8 \mathrm{GHz}$. Nuand supplies and application programming interface that can be used with several popular languages such as $\mathrm{C} / \mathrm{C}++$ and Python. However, an open source tool called GNURadio offers an intuitive flowchart design process that allows engineers to more easily implement digital signal processing techniques, such as digital filters and demodulators [19]. GNURadio takes care of many more complicated back-end processes 
such as threading, allowing a high level of abstraction for the design process while still allowing for high performance digital signal processing programs.

2. Miniaturization of an interrogator using off-the-shelf (COTS) components mounted on a $P C B$

The prior signal conditioning circuit of the interrogator involves many SMA cableconnected COTS components from the manufacturer Mini-Circuits. To make this system more compact, allowing it to be mounted in a confined space or small unmanned aerial vehicle (UAV) this system must be much more lightweight. This can be done by using COTS surface mount components. Designing the PCB to mount these surface mount components can be more complicated, but it will offer a much more convenient solution.

3. Design of a harmonic transponder using minimal lumped element components

The final stage of this work is to develop a new harmonic transponder. The main goal of this harmonic transponder will be to minimize the need for external lumped element components. A simple, robust device will be designed on a single substrate, only needing a single external diode to complete the device. A novel sensing technique will also be explored as future work, using a microstrip technology known as an interdigital capacitor.

\subsection{Thesis Layout}

A background and history of harmonic transponders, as well as a introduction to SDR will be given in Chapter 2. Chapter 3 will discuss the design process of the 
harmonic transponder designed for this work, and will provide simulations of the the necessary components of the harmonic transponder. Chapter 4 will go over the SDR interrogator, including the digital signal processing for interrogation, and the external filter and amplifier board used to minimize the setup. Chapter 5 will go over measurement results using the entire harmonic transponder system, that being the interrogation system designed in Chapter 4 and the harmonic transponder in Chapter 3. Finally, Chapter 6 concludes the thesis with a discussion about the results of this work and future work. 


\section{CHAPTER 2}

\section{BACKGROUND}

This chapter will cover design and applications of harmonic transponders. An introduction to SDR will be covered as well. As mentioned in Chapter 1, the proposed interrogator's main component will be a SDR, so it is important that the main ideas are covered before delving into the design.

\subsection{Passive Transponder Technologies}

RFID tags have been used extensively since the 1990's for toll collection, traffic management, controlling access to buildings, and several other applications [20]. These devices operate at a single frequency utilizing back-scatter wireless communications. Energy is harvested from the interrogation signal, modulated with electronically stored information, and then reflected back to an interrogator. In contrast, passive harmonic transponders take advantage of non-linearities in circuit elements (e.g., diodes) allowing for multi-band operation. 


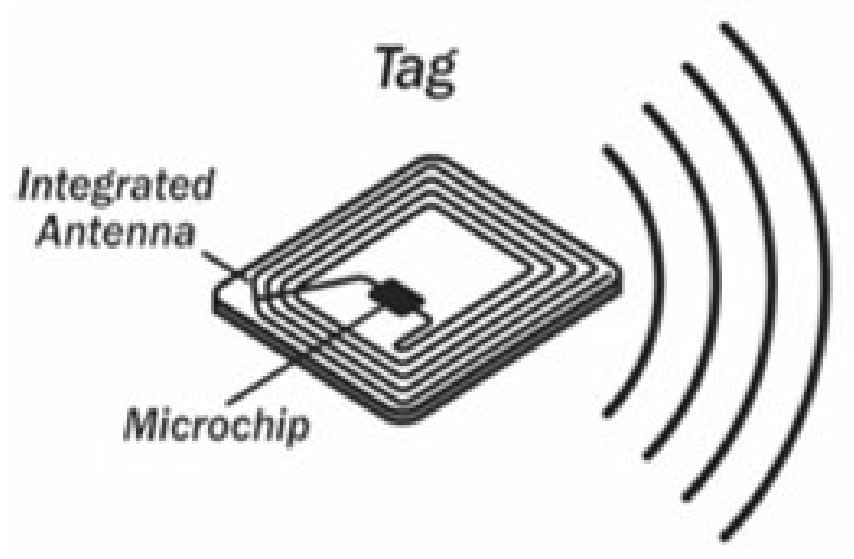

Figure 2.1: A general RFID tag. The microchip is responsible for modulating back-scattered information with digital data encoded in the chip. Image from [1]

\subsubsection{RFID}

RFID tags are often used for, as their name indicates, identification of objects. They often find use cases in manufactured goods and materials allowing for identification of objects at a distance, and offering more range and ability than older systems, such as the earlier bar code system [21]. RFID tags come in a range of shapes, sizes, and capabilities.

These tags often have a single antenna which is responsible for both receiving the interrogation signal, and transmitting electromagnetic energy back to the interrogator. The electromagnetic waves from the interrogation system provide enough energy to power the integrated silicon microchip on the tag, of which a diagram of can be found in Figure 2.1. The microchip then modulates the received energy with digitally encoded information stored in memory on the tag, and transmits that information back to the interrogator using the same antenna. In some chips, the energizing signal can also carry commands to flash new information on the tag [22]. RFID tags are 


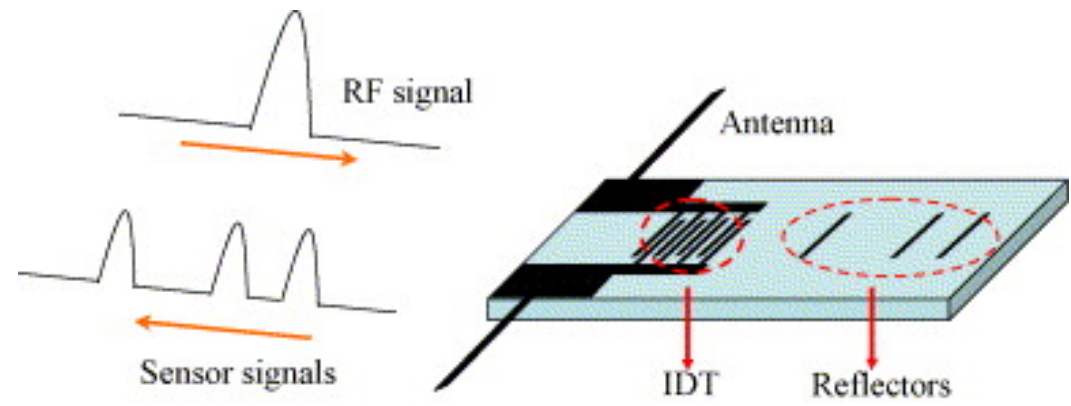

Figure 2.2: Diagram of a typical SAW tag. These devices modulate electromagnetic energy using an acoustic medium.

often used in the ultra high frequency (UHF) band at $433 \mathrm{MHz}$, or $900 \mathrm{MHz}$ [23]

\subsubsection{SAW}

SAW devices are another solution to passive wireless sensing. These devices often consist of a dipole antenna connected to an interdigital transducer (IDT) which converts electromagnetic energy from an interrogator to acoustic energy, which then propagates through a piezoelectric substrate. Reflectors on the tag reflect back acoustic energy to the IDT which produced it.

To illustrate how a SAW devices can be used as a sensor, we will give an example of how a SAW pressure sensor would work. When a SAW sensor (such as the one shown in Figure 2.2 is compressed, the phase velocity of the piezoelectric substrate will increase. In turn, this will increase the delay between the return pulses made by the reflector. A larger delays between the return pulses allow the interrogator to distinguish between different measured pressures.

Similar to RFID, SAW devices utilize the same frequency for interrogation and return. Both devices use an interrogation signal, and wait for a response from the passive device. In the next Section, harmonic transponders will be introduced. 


\subsubsection{HARMONIC TRANSPONDERS}

As introduced in the Chapter 1, harmonic transponders rely on non-linear circuit elements to generate harmonics in an interrogation signal. These non-linear elements are often Schottkey diodes, which perform the actual frequency multiplication step from the fundamental frequency $\left(f_{0}\right)$ to the harmonic frequency $\left(2 \times f_{0}\right)$. It is important for the non-linear element to convert $f_{0}$ to $2 \times f_{0}$ as efficiently as possible.

The other main component of a harmonic transponder are the antennas (or in some cases, a singular antenna). The antennas are responsible for the communication between the harmonic transponder and the interrogator. It is important that the antenna both resonates and is matched to the non-linear element at both $f_{0}$ and $2 \times f_{0}$ to maximize the transponder's ability to reflect information back to the interrogator.

As mentioned above, harmonic transponders differ from SAW and RFID in the fact that they use the harmonic of the interrogation signal for the return. This is advantageous for simplifying the interrogator, since the interrogator can be generating an interrogation signal at one frequency, while simultaneously measuring the return signal at a second frequency. Furthermore, because interrogation and return are two distinct frequencies, self interference (i.e., interrogation disrupting the return) is

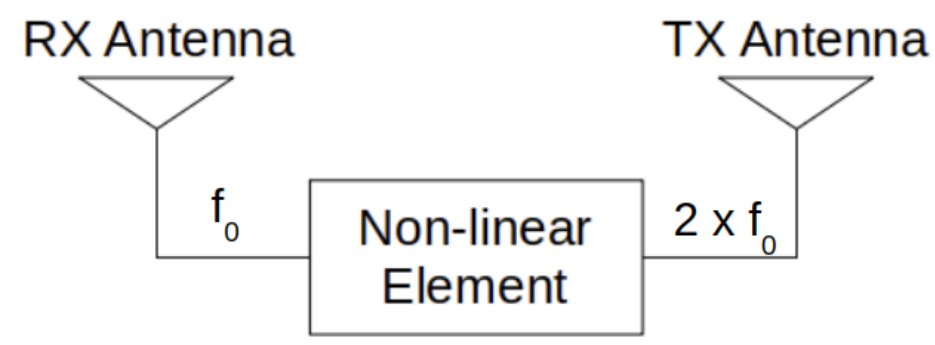

Figure 2.3: General harmonic transponder layout 
eliminated completely.

\subsection{Harmonic Radar Systems}

Harmonic transponders have numerous applications. Specifically, they can be quite effective in cluttered environments. In an environment with large amounts of clutter, transponders that respond with the same frequency of interrogation (such as traditional RFID) would experience large amounts of reflections causing interference in the return signal. The harmonic of the interrogation signal is much easier to pick up with a receiver, seeing as typical objects (e.g., floors, trees, etc.) do not have a harmonic response when hit with RF energy. A typical harmonic transponder circuit layout can be found in Figure 2.3.

Because of this unique property of harmonic transponders, these devices have been used to track small creatures in biological studies [17]. The tags used for this purpose consist of just a single diode soldered to short leads allowing for a form-factor compact enough to be mounted on these creatures without disturbing their natural behavior.

Other designs of harmonic transponders find applications in passive sensor systems, offering higher performance in cluttered environments over single-frequency RFID [24]. The sensing ability of some harmonic transponders operate solely on received power characteristics, as such in $[14,25]$. Other harmonic transponders contain a sensing element which (often capacitive) alters the resonant frequency in the tag.

Perhaps the most well known harmonic radar system (to snow sports enthusiasts), would be the RECCO Rescue System [26]. The RECCO system had been responsible 


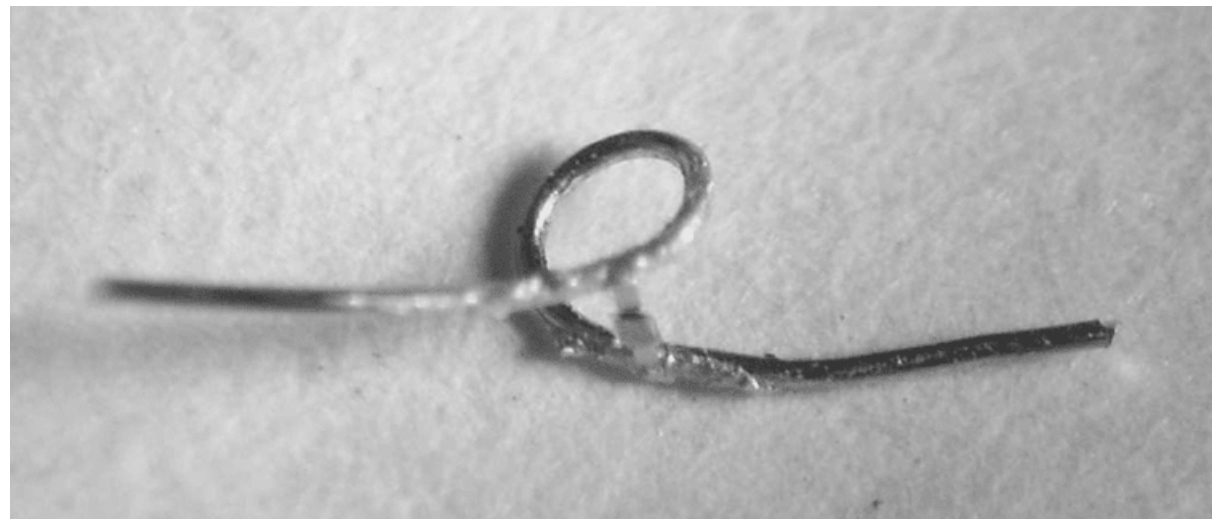

Figure 2.4: Example of loop harmonic transponder from [2]. Loop diameter is $1 \mathrm{~mm}$.

for saving the lives of many outdoor enthusiasts without traditional beacon equipment. RECCO tags are very small and passive, allowing them to be mounted on the outside of various consumer outdoor gear. This particular harmonic radar system uses a fundamental frequency of $866 \mathrm{MHz}$, allowing the interrogation and return signals to easily penetrate several feet of snow. Various other applications of harmonic transponders and harmonic radar systems will be discussed in the sections below.

\subsubsection{Small Creature Tracking}

Applications in harmonic transponders have found a large niche in tracking small creatures, dating back to the 1980's [27]. Tracking small creatures (such as insects) with traditional radar is nearly impossible due to their minuscule radar cross-section. To increase the visibility of these animals in the RF spectrum, harmonic radar tags are attached to the creature, increasing the visibility of the creature drastically to the interrogator.

Harmonic radar tags for small creatures are often designed to be quite small. In [28], the authors designed tags to operate at millimeter-wave frequencies (mmWave), 
those being a $38.5 \mathrm{GHz}$ fundamental frequency, and a $77 \mathrm{GHz}$ harmonic frequency. This allowed the antennas on the device to be extremely compact, resulting in a tag size of only $5.15 \mathrm{~mm} \times 2.91 \mathrm{~mm}$.

Other harmonic radar tags are much simpler, such as those in $[2,16]$. These tags consist solely of some wire and a single Schottky diode, as seen in Figure 2.4. The systems in $[2,16]$ both use quite high interrogation fundamental frequencies frequencies, those being $9.41 \mathrm{GHz}$ and $5.8 \mathrm{GHz}$ respectively. For the application of animal tracking, often a range measurement is desired.

When harmonic transponders are paired with a well-designed harmonic radar interrogator, these systems can easily outperform RFID systems with similar attributes. [29] demonstrated that insects tagged with a harmonic transponder could be detected up to $58 \mathrm{~m}$ away at an effective isotropically radiated power (EIRP) of only $42 \mathrm{dBm}$, significantly farther than similar RFID systems.

Recent work in harmonic radar shows that tagged insects can be detected at close to $500 \mathrm{~m}$ using a high power harmonic radar setup (1 kW) [17]. Work in [17] use the familiar single diode transponder, similar to the one seen in Figure 2.4. This system also operates at quite high frequencies $(9.4 \mathrm{GHz} / 18.8 \mathrm{GHz})$, and uses advanced radar techniques for ranging and angle-of-arrival calculations.

\subsubsection{SEnsing And Harsh Environments}

Lower frequency systems (below $5 \mathrm{GHz}$ ) for sensing in harsh environments has also been explored by many. Work in [24] has shown that using harmonic transponders can significantly reduce multi-path interference for indoor ranging at $1.14 \mathrm{GHz} / 2.28$ GHz. Multi-path conditions are caused by interference of reflected electro-magnetic 
waves. Indoor environments typically have quite high multi-path components since there are many things for the electro-magnetic signal to reflect off (e.g., walls, ceilings, computers, etc.). Better multi-path performance is due to the fact that the return signal from a harmonic transponder (i.e., two times the interrogation frequency) does not interfere with the interrogation signal. This can also prove to be beneficial for pipe localization, reducing the need for expensive ground penetrating radar equipment [25].

Other work shows authors adding sensing elements to harmonic transponders $[18,30]$ at $2.5 \mathrm{GHz} / 5 \mathrm{GHz}$. Often times, sensors are integrated into harmonic transponders using a capacitive element. A change in capacitance in the circuit will change the frequency response of the tag, allowing the interrogator to interpret a change in the frequency response as a change in the medium being sensed. [30] leveraged a microfluidic-capacitive gap to modulate the response of the sensor tag.

Work in [18] uses a metamaterial-inspired antenna, which can hold a liquid sample. The frequency of operation in [18] is $902-928 \mathrm{MHz} / 1.856 \mathrm{GHz}$. The transmit antenna is a $3-\mathrm{D}$ structure that consists of a monopole wound in a helical pattern. This transponder operates of the same principal many harmonic transponder sensors do. As liquid is added to the antenna structure, the inductor-capacitor (LC) resonance of the actual device will change. Changes in this LC resonance then correspond to a peak shift of a frequency swept signal.

\subsection{Frequency Doubling Reflectenna}

This work considers a particular harmonic transponder, referred to as a frequency doubling reflectenna (FDR). A distinct advantage that the FDR has over typical 
RFID is its low activation power. Past work has shown that the minimum activation power of the FDR is on the order of $-30 \mathrm{dBm}$, while the minimum activation power of typical RFID is around $-14 \mathrm{dBm}[3]$. This implies that the maximum sensing range of the FDR is greater than that of RFID and/or a lower power interrogator can be developed for this device.

The FDR is a resonant circuit that when interrogated at frequency $f_{1}$, re-radiates twice that frequency, $f_{2}=2 \times f_{1}$ which was originally presented in [3]. The particular device utilized in this research operates at $f_{1}=1.28 \mathrm{GHz}$ to produce a $f_{2}=2.56$ $\mathrm{GHz}$ back-scattered signal. This frequency doubling effect is achieved by placing a Schottky diode between the transmit and receive elements, partially rectifying the received signal. This introduces several harmonics in the device, which are then reflected back through the transmit element. Figure 2.5 shows the layout of the particular FDR used in this work.

Key attributes of the FDR include a 1\% conversion efficiency at -30 dBm input power (20 dB conversion loss) and a compact form factor due to the two quarterwavelength patch antennas [3]. Applications of this FDR device were explored in [14], where it was used to investigate in situ monitoring of soil characteristics.

\subsection{An Introduction to Software De- FINED RADIO}

Radio systems have evolved with modern advances in wireless communications and computing. In 1864, James Clerk Maxwell showed through a series of mathematical proofs that electromagnetic wave could propagate though free space [31]. Since then, 


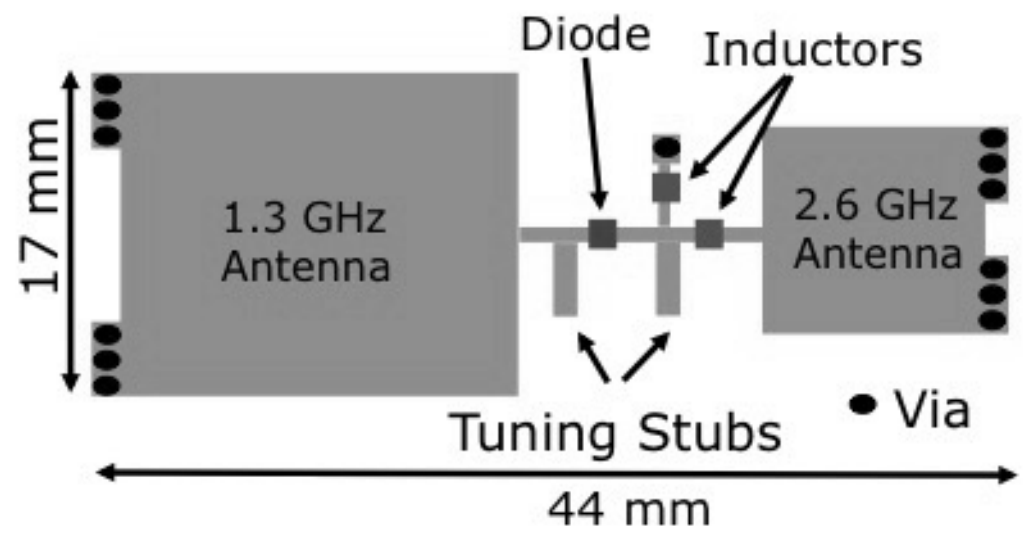

Figure 2.5: Layout of frequency doubling reflectenna (FDR) from [3]

engineers, scientists, and business-people have been exploring and innovating in the field of wireless communications.

Traditionally, wireless communications have been implemented using hardware, such as analog demodulators. A simple example of this is the AM radio demodulator, which can be implemented using a simple diode, capacitor, and antenna circuit [32]. Software defined radio (SDR) offers a different approach, where tasks such as demodulation can be performed in software using digital signal processing techniques.

The SDR Forum, working in collaboration with the Institute of Electrical and Electronic Engineers (IEEE) P1900.1 group has set a standard definition for SDR, that being "Radio in which some or all of the physical layer functions are software defined".

The range of commercially available SDRs varies. Some are silicon chips that simply provide an interface from the RF spectrum to baseband (i.e., $0 \mathrm{~Hz}$ ), such as the Analog Devices AD9361 [33]. Other SDRs offer a fully functional radio, equipped with powerful application programming interfaces (API) allowing the user to design entire communications systems with a single board, an example being the Nuand 


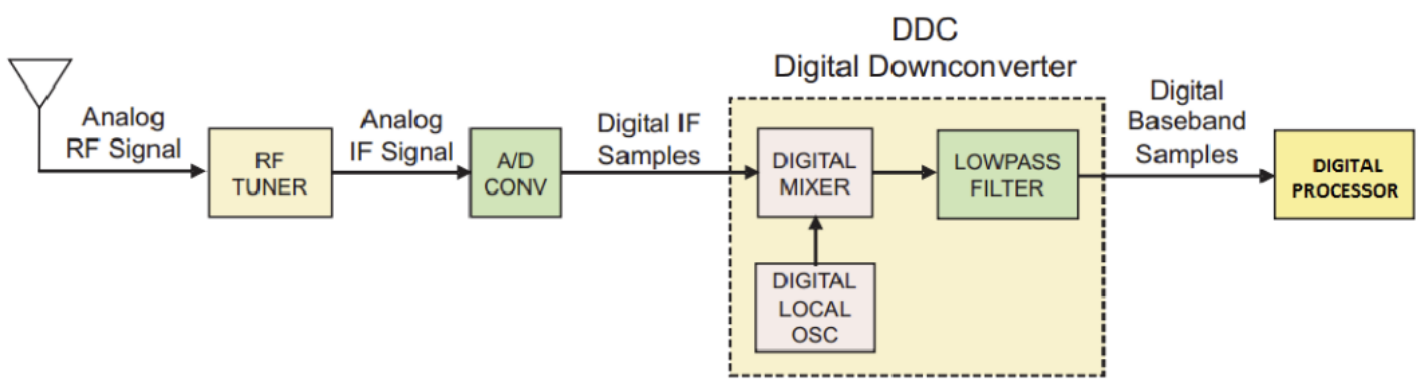

Figure 2.6: Typical SDR Harware [4]

BladeRF [15].

\subsubsection{Typical HaRdWARE IN SDR}

The most pure form of a SDR would simply be an antenna connected to a digital to analog converter. This architecture for SDR can be quite challenging to implement, since analog-to-digital converters with several $\mathrm{GHz}$ bandwidth are very difficult to design. In practice, an antenna is often connected to a flexible RF hardware, where the RF signal is the down-converted to baseband (i.e., $0 \mathrm{~Hz}$ ). The signal is downconverted into inphase-quadrature data (IQ), which is a complex value. This downconverted signal is then low pass filtered to remove any out-of-band energy that would cause aliasing once the signal is sampled, since the Nyquist rate must be satisfied. The Nyquist sampling theorem can be found in Equation 2.1, where $f_{s}$ is the sampling rate of the system, and $B$ is the bandwidth of the signal of interest.

$$
f_{s} \geq 2 B
$$

Once the down-converted RF signal has been sampled into IQ data the samples 
are then sent to a digital signal processing (DSP) medium, such as a host PC or FPGA. Most consumer SDRs often combine multiple DSP mediums. For example, the Nuand BladeRF uses both and FPGA for some DSP and data scaling, then sends the processed samples over USB 3.0 to a host PC [15]. A diagram of some typical SDR hardware can be found in Figure 2.6

\subsubsection{The Nuand BladeRF SDR}

The SDR used in this work is the Nuand BladeRFx40 [15]. The BladeRF is a highly capable, low cost SDR, capable of tuning anywhere within the band of $300 \mathrm{MHz}$ to 3.8 GHz. It is capable of full-duplex operation with a maximum sampling rate of 40 mega-samples per second (MSPS) of 12-bit IQ samples, each I and Q sample is represented with 6 bits. The instantaneous bandwidth (IBW) of the BladeRF is 28 $\mathrm{MHz}$, meaning that the maximum bandwidth RF signal that can be viewed with the BladeRF is $28 \mathrm{MHz}$.

A key attribute of the BladeRF is it's full-duplex ability. Full-duplex means that the SDR is capable of both transmitting and receiving signals at the same time. The system designed in this work could be made much more compact by combining both transmit and receive operations into a single board. Most other systems, like those mentioned in Section 2.2, often use completely separate transmit and receive chains for interrogating harmonic transponder making them quite large.

The BladeRF has two separate phase locked loops (PLLs) allowing the transmit and receive chains to be tuned to two different frequencies. For the application in this work, the transmit chain will be tuned to the interrogation frequency $\left(f_{1}\right)$ and the receive chain to the return from the harmonic transponder $\left(2 \times f_{1}\right)$. 


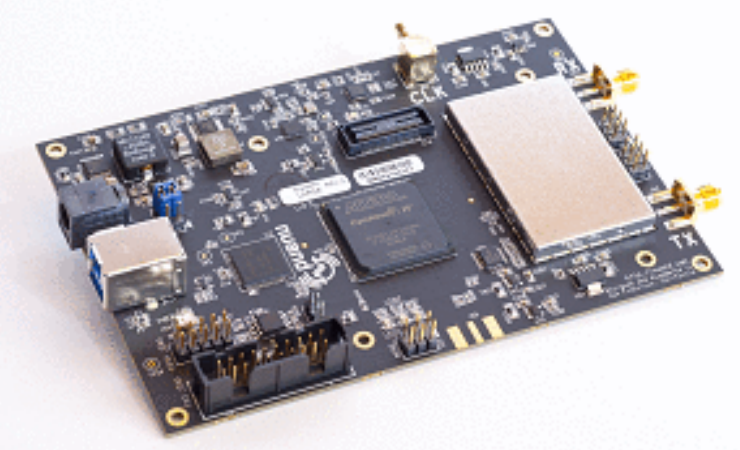

Figure 2.7: Nuand Bladerfx40 SDR

\subsubsection{GNU RADIO}

The open source platform, GNU Radio, has become increasingly more popular in many industries such as military, and academia as a method for programming commercial SDRs [19]. GNU Radio allows a user to create intuitive signal processing flowgraphs. Various blocks in GNU Radio include digital filters, various demodulators, mixers, discrete math operations, and many others.

Since GNU Radio is an open source project, any developer is allowed to contribute. Dimitri Stolnikov is the author of the GNU Radio package called Osmocom [34]. The Osmocom project provides interfaces for several popular SDRs on the market. The Osmocom sink and source blocks are responsible for connecting the user's hardware to the GNU radio application. The source block is responsible for relaying IQ samples to other blocks, while the sink block sends IQ data to the SDR to be transmitted. 


\begin{tabular}{|c|c|}
\hline $\begin{array}{l}\text { Signal Source } \\
\text { Sample Rate: } 20 \mathrm{M} \\
\text { Waveform: Cosine } \\
\text { Frequency: } 1 \mathrm{k} \\
\text { Amplitude: } 1 \\
\text { Offset: } 0\end{array}$ & $\begin{array}{l}\quad \text { osmocom Sink } \\
\text { Sample Rate (sps): } 20 \mathrm{M} \\
\text { Ch0: Frequency (Hz): } 100 \mathrm{M} \\
\text { Ch0: Freq. Corr. (ppm): } 0 \\
\text { Ch0: RF Gain (dB): } 10 \\
\text { Ch0: IF Gain (dB): } 20 \\
\text { Ch0: BB Gain (dB): } 20\end{array}$ \\
\hline
\end{tabular}

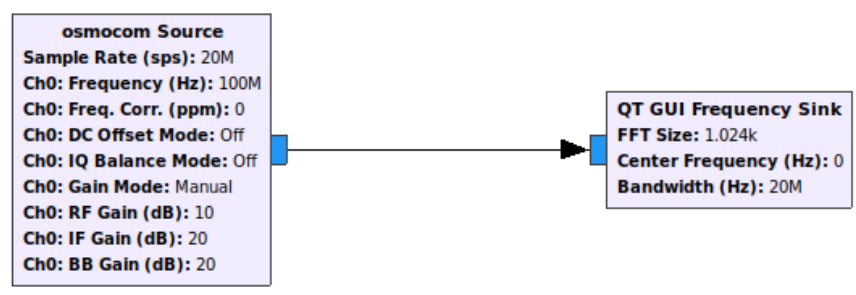

Figure 2.8: Example of a GNU Radio flow graph

\subsection{SDR FOR SPECTRAL ANALYsis}

Using SDR for spectral analysis differs from using a spectrum analyzer. Because the SDR samples signals in the time domain with an analog-to digital converter, we must use the discrete Fourier transform (DFT) to view the signal in the frequency domain. For some discrete time signal $x[n]$ of length $N$ sampled at some frequency $f_{s}$, we can write the discrete Fourier transform as:

$$
X[k]=\sum_{n=0}^{N-1} x[n] e^{-j 2 \pi k n / N}
$$

Where $X[k]$ is the DFT. The frequency of the DFT at an index can be found using the following equation:

$$
f_{k}=\frac{f_{s}}{N} k
$$


Where $-\frac{N}{2} \geq k \geq \frac{N}{2}-1$.

The intrinsic assumption of the DFT is that $x[n]$ is periodic for all time. When it comes to processing real world signals, it is safe to say that signal is almost never periodic for all time. It is also safe to assume that there will be discontinuities in your sampled signal. For example, the DFT of a single period of a perfect sinusoid will look like a perfect impulse in the frequency domain. If a signal is not perfectly periodic (like most real-world signals) a window is used to mitigate an effect known as spectral leakage [35]. Many windows have been made over the years, but the Blackman-Harris window remains to be the most common window used for reducing spectral leakage in spectral analysis. The coefficients for the Blackman-Harris window can be calculated using the following equation:

$$
\begin{array}{r}
w[n]=a_{0}-a_{1} \cos \left(\frac{2 \pi n}{N}\right)+a_{2} \cos \left(\frac{4 \pi n}{N}\right)-a_{3} \cos \left(\frac{6 \pi n}{N}\right) \\
a_{0}=0.35875 ; \quad a_{1}=0.48829 ; \quad a_{2}=0.14128 ; \quad a_{3}=0.01168
\end{array}
$$

Where $N$ is the length of the window, and all the coefficients are constants of the window.

Figure 2.9 illustrates the effect a window has on a sinusoidal signal. Discontinuities in the sinusoid (i.e., the signal not being perfectly periodic) cause the power of the sinusoid to be spread into high frequency bins in it's DFT. It is apparent in Figure 2.9 that the power has been concentrated in the lower frequency bins of the DFT of the windowed signal, which more accurately represents the frequency domain of the signal of interest. 

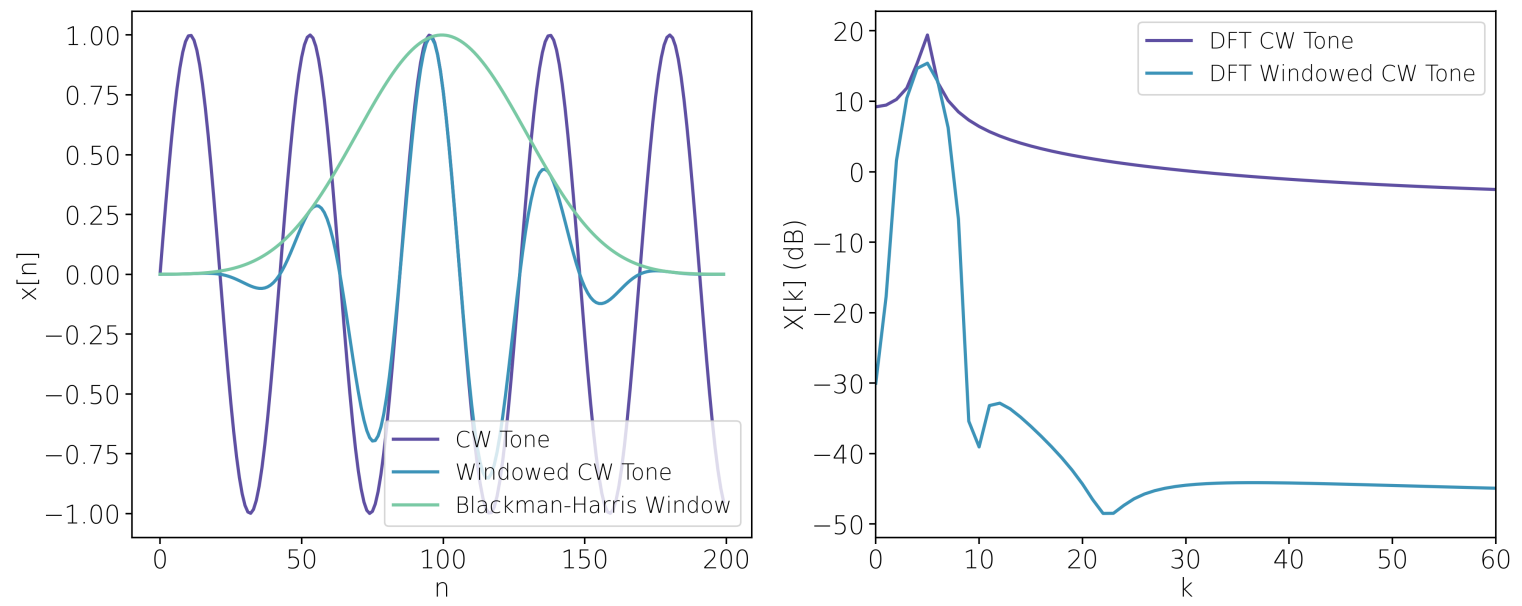

Figure 2.9: (L) Time domain. Continuous wave $(C W)$ tone with and without window applied. (R) Frequency domain. DFT of $C W$ tone with and without window applied.

\subsection{Conclusion}

In this chapter, prior literature on harmonic transponders and an introduction to software defined radio was presented. Harmonic transponders have a wide range of applications, ranging from insect and small creature tracking to sensing in harsh environments. Interrogators for harmonic transponders are often large and bulky, due to the power requirements for some applications.

Software defined radio was introduced as a solution to harmonic transponder interrogation. Full-duplex SDRs can be capable of transmitting information in one frequency band and receiving information in another. According to the authors best knowledge, SDR has not been leveraged as an interrogator for harmonic transponders. SDR could offer a flexible alternative to the traditional signal generator and receiver combination. In addition, several interrogation schemes could be investigated in future work. 


\section{CHAPTER 3}

\section{HaRmonic Transponder Design}

This Chapter will go over the design and simulation of a harmonic transponder with a fundemental frequency of about $1.3 \mathrm{GHz}$, similar to the FDR presented in Section 2.3. First, the design of two patch antennas will be discussed. These patch antennas

must be tuned to the interrogation frequency $\left(f_{1}\right)$ and the return frequency $\left(2 \times f_{1}\right)$, respectively. Matching topologies for integrating the antenna and diode doubler will also be presented. This harmonic transponder was manufactured here at UVM in the High Performance Circuits Laboratory on a printed circuit board milling machine.

\subsection{Patch Antennas}

Due to the constraints of being limited to a printed circuit board, the antennas must be able to be printed onto a substrate. Patch antennas can easily be milled onto a $\mathrm{PCB}$, and still maintain good gain and directivity properties. While some transponder design use a single antenna, this design will use two separate antennas tuned to resonate at frequencies $f_{1}$ and $2 \times f_{1}$, and will be placed on each side of the 


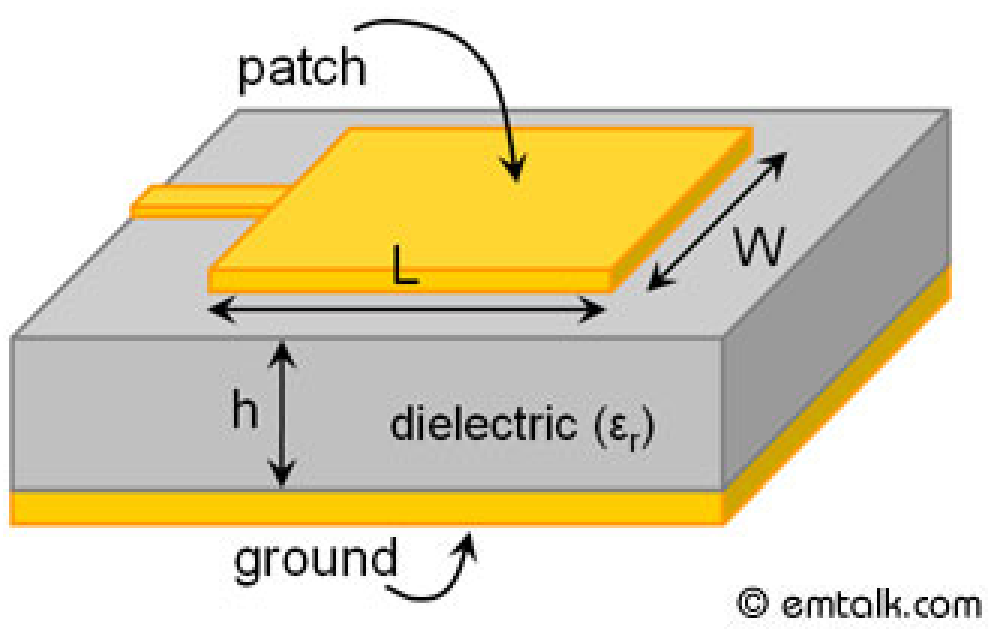

Figure 3.1: Dimensions of a typical patch antenna

diode doubler.

\subsubsection{Design}

Patch antennas are designed to resonate at specific frequencies based on the length and width of the antenna. The following equations below are used to calculate the resonant length and width of a patch antenna [36].

First, to calculate the antenna width, we use Equation 3.1. Where $c$ is the speed of light, $f_{0}$ is the desired frequency of operation, and $\epsilon_{r}$ is the relative dielectric constant of the substrate. In our case, the relative dielectric constant of the Rogers RO4350B board is $\epsilon_{r}=3.66[37]$.

$$
W=\frac{c}{2 f_{0} \sqrt{\frac{\epsilon_{r}+1}{2}}}
$$

To calculate the effective dielectric constant, we use Equation3.2, which will be 
used to calculate the proper length of the antenna.

$$
\epsilon_{e f f}=\frac{\epsilon_{r}+1}{2}+\frac{\epsilon_{r}-1}{2} \frac{1}{\sqrt{1+12 \frac{h}{W}}}
$$

Next, the effective length must be calculated to find the length extension.

$$
\begin{gathered}
L_{e f f}=\frac{c}{2 f_{0} \sqrt{\epsilon_{e f f}}} \\
\Delta L=0.412 h \frac{\left(\epsilon_{e f f}+0.3\right)\left(\frac{W}{h}+0.264\right)}{\left.\epsilon_{e f f}-0.258\right)\left(\frac{W}{h}+0.8\right)}
\end{gathered}
$$

Finally, we can calculate the length of the patch antenna.

$$
L=L_{e f f}-2 \Delta L
$$

We can now use this process to design both patch antennas for the harmonic transponder

\subsubsection{Final Patch Antennas}

After using the equations from Section 3.1.1, the patch antennas can be simulated using an RF simulation software. In this case, Keysight Advanced Design System was used. The resultant $1.3 \mathrm{GHz}$ antenna dimensions were calculated to be $75.5 \mathrm{~mm}$ x 60 $\mathrm{mm}$. Likewise, the $2.6 \mathrm{GHz}$ antenna dimensions were found to be $37.7 \mathrm{~mm}$ x $30 \mathrm{~mm}$.

For simplicity of design, each patch antenna was designed to have an approximate input impedance of $50 \Omega$, since we are using $50 \Omega$ transmission lines in the design. This is done by using an inset-feed patch antenna. Typically, the input impedance 


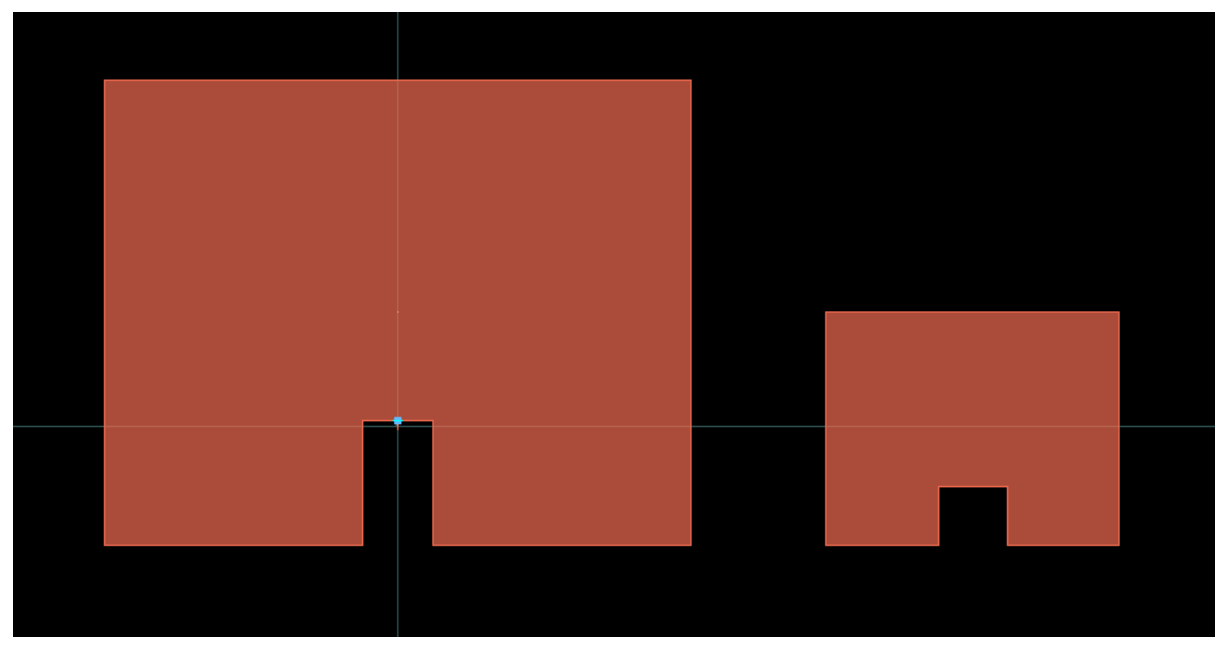

Figure 3.2: Final antennas designs. $1.3 \mathrm{GHz}$ antenna $(75.5 \mathrm{~mm} \times 60 \mathrm{~mm})$ is on the left, and $2.6 \mathrm{GHz}$ antenna $(37.7 \mathrm{~mm} \times 30 \mathrm{~mm})$ is on the right.

of a patch antenna is around $300 \Omega$ at its edge, but by adding an inset to the patch antenna the input impedance can be reduced to $50 \Omega$ [38].

Using an ADS electromagnetic simulation, the 1.3 GHz was found to have a gain and directivity of $4.6 \mathrm{~dB}$ and $6.6 \mathrm{~dB}$, respectively. Similarly, the $2.6 \mathrm{GHz}$ antenna have a gain and directivity of $5.17 \mathrm{~dB}$ and $6.17 \mathrm{~dB}$, respectively.

\subsection{Diode Doubler}

The main element of a harmonic transponder is the diode doubler, the part of the device that induces harmonics in the fundamental frequency. Diode spice models were obtained from the manufacturer which can be found in Table 3.1. The diode used is the Skyworks SMS7630 Schottky Diode [39]. To correctly model the diode for the doubler, parasitics of the package must be incorporated. For the SC-79 package, there is a lead inductance of $0.6 \mathrm{nH}$, and a package capacitance of $0.1 \mathrm{pF}$. This diode model 


\begin{tabular}{|c|c|c|}
\hline Parameter & Units & SMS7630 \\
\hline $\mathrm{I}_{S}$ & $\mathrm{~A}$ & $5 \mathrm{E}-6$ \\
$\mathrm{R}_{S}$ & $\Omega$ & 20 \\
$\mathrm{~N}$ & - & 1.05 \\
$\mathrm{TT}$ & $\mathrm{sec}$ & $1 \mathrm{E}-11$ \\
$\mathrm{C}_{J O}$ & $\mathrm{pF}$ & 0.14 \\
$\mathrm{M}$ & - & 0.40 \\
$\mathrm{E}_{G}$ & $\mathrm{eV}$ & 0.69 \\
$\mathrm{XTI}$ & - & 2 \\
$\mathrm{~F}_{C}$ & - & 0.5 \\
$\mathrm{~B}_{V}$ & $\mathrm{~V}$ & 2 \\
$\mathrm{I}_{B V}$ & $\mathrm{~A}$ & $1 \mathrm{E}-4$ \\
$\mathrm{~V}_{J}$ & $\mathrm{~V}$ & 0.34 \\
\hline
\end{tabular}

Table 3.1: SPICE parameters of Skyworks SMS7630

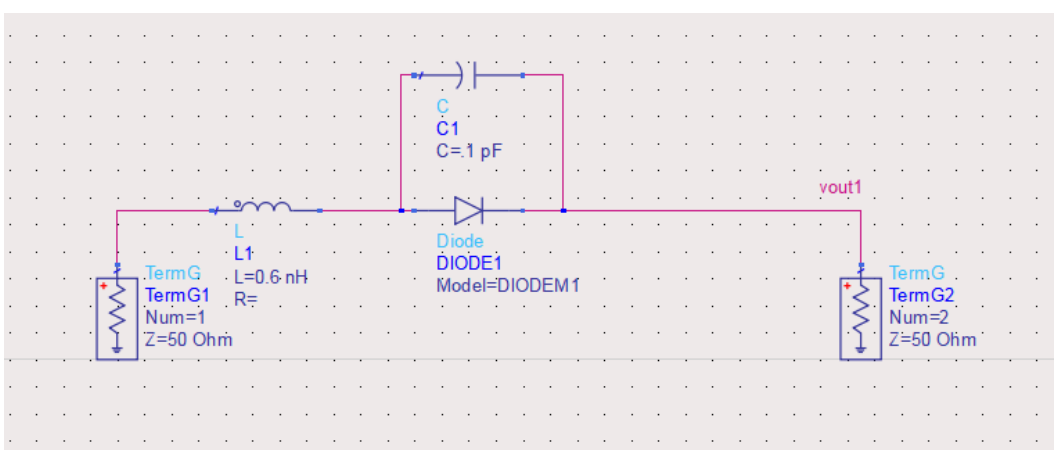

Figure 3.3: Diode model for Skyworks SMS7630 with SC-79 package. Capacitor and inductor are parasitics of the $S C$-79 package.

was used to generate the input and output impedances over a range of frequencies. The circuit diagram for the diode model used can be found in Figure 3.3.

\subsubsection{Doubler Impedance}

To maximize power transfer, the antenna impedance must be matched to the input and output impedance's of the diode doubler. Often, antenna matching to the diode doubler is done by directly modifying the impedance of the antenna. This is usually 


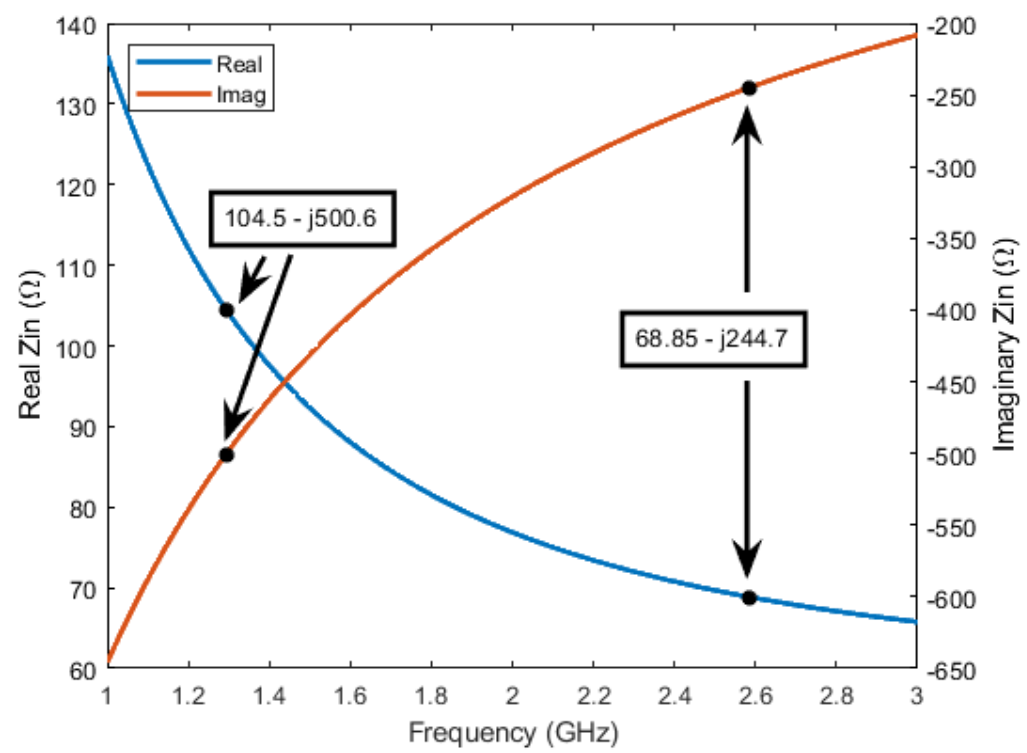

Figure 3.4: Input (1.293 GHz) and output (2.586 GHz) diode doubler impedances

done by optimizing the size or shape of the antennas $[3,25,40]$.

Other approaches involve using external matching circuits as such in [41], where discrete components were used to match the impedance of the diode doubler to a single antenna. This approach allows the antenna design to be straight forward, but it can be difficult to find good performance using lumped element components since they only come discrete values only.

In this work, standard patch antennas are matched to the diode doubler impedances using transmission lines. This allows for optimal patch antenna sizing, while still maintaining good matching performance on the board. The real an imaginary impedance of the diode doubler can be found in Figure 3.4. At the interrogation frequency (1.293

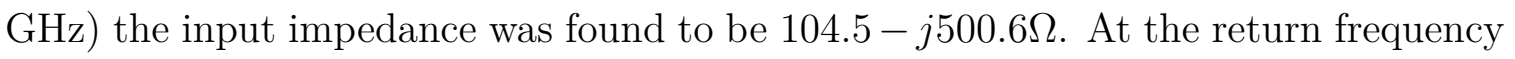
$(2.586 \mathrm{GHz})$ the output impedance was calculated to be $68.85-j 244.7 \Omega$. 


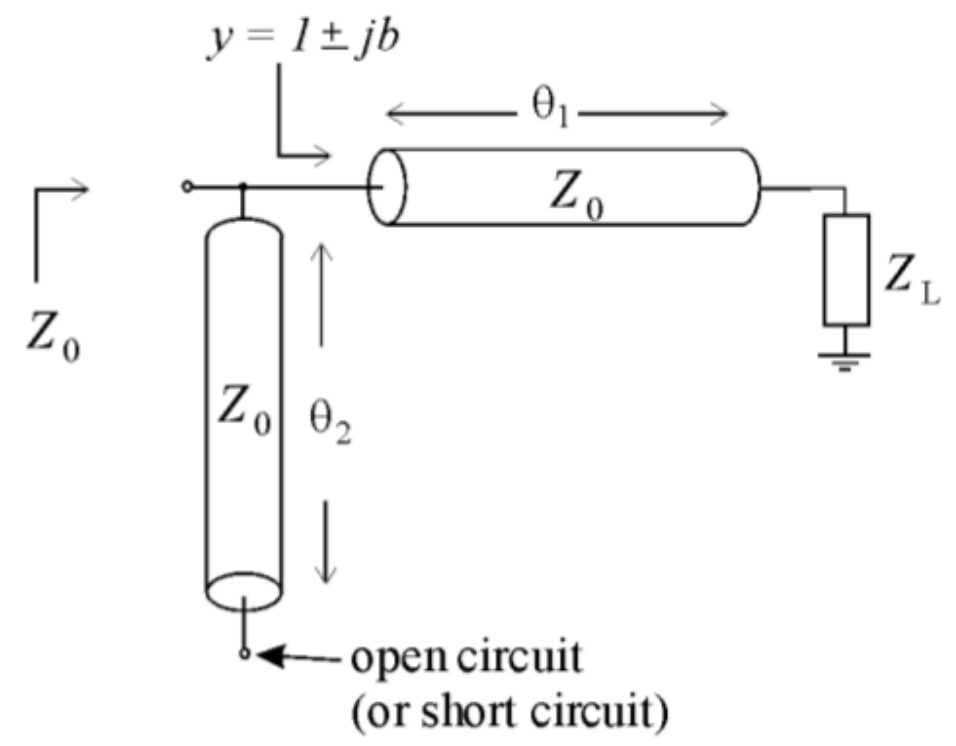

Figure 3.5: Diagram of single stub matching network

\subsubsection{MATCHING}

A single stub matching technique is used to match both antennas to the input and output of the diode doubler. Single stub matching is often used to match real to complex impedance's [42]. The impedance's of each antenna are real (50 $\Omega$ ), and the input and output impedance of the diode doubler are complex, making our harmonic transponder a good candidate for single stub matching. First, we must analytically find the length of the series transmission line.

The input impedance of a lossless transmission line of length $d$ of characteristic

impedance $Z_{0}$ terminated by an load impedance, $Z_{L}$ can be found in Equation3.6, 
where $\beta$ is the propagation constant.

$$
Z_{I N}=Z_{0} \frac{Z_{L}+j Z_{0} \tan (\beta d)}{Z_{0}+j Z_{L} \tan (\beta d)}
$$

Next we set $Z_{0}$ equal to the source impedance, $R_{S}$, and $\tan (\beta d)$ equal to some constant $t$, to be found shortly. The input admittance can then be written as the following equation.

$$
Y_{I N}=\frac{1}{Z_{I N}}=\frac{R_{S}+j Z_{L} t}{R_{S}\left(Z_{L}+j R_{S} t\right)}=G_{I N}+j B_{I N}
$$

Where $G_{I N}$ and $B_{I N}$ are the real and imaginary parts of the input admittance, respectfully. Now, after substituting the normalized load impedance $Z_{L} / R_{S}=r+j x$, and separating Equation3.7 into its real and imaginary parts, we get:

$$
\begin{gathered}
G_{I N}=\frac{r\left(1+t^{2}\right)}{R_{S}\left(r^{2}+x^{2}+t^{2}+2 x t\right)} \\
B_{I N}=\frac{x t^{2}+\left(r^{2}+x^{2}-1\right) t+x}{R_{S}\left(r^{2}+x^{2}+t^{2}+2 x t\right)}
\end{gathered}
$$

Next, we must find $d$, which implies $t$. By setting the input conductance, Equation3.8, equal to the source conductance, we can then solve for $t$.

$$
\frac{r\left(1+t^{2}\right)}{R_{S}\left(r^{2}+x^{2}+t^{2}+2 x t\right)}=\frac{1}{R_{S}}
$$

Equation 3.10 can then be rearranged to be:

$$
(r-1) t^{2}-2 x t-\left(r^{2}+x^{2}-r\right)=0
$$


The quadratic formula can then be used to find the two solutions to Equation3.11.

$$
\begin{aligned}
& t_{1}=\frac{x+\sqrt{r\left(r^{2}+x^{2}-2 r+1\right.}}{r-1} \\
& t_{2}=\frac{x-\sqrt{r\left(r^{2}+x^{2}-2 r+1\right.}}{r-1}
\end{aligned}
$$

Since $\tan (\beta d)=t$, and $\beta=2 \pi$, we can then find the two electrical lengths of the series transmission lines specified in radians.

$$
\begin{aligned}
& d_{1}=\frac{\lambda}{2 \pi} \tan ^{-1}\left(t_{1}\right), \quad t_{1} \geq 0 \\
& d_{2}=\frac{\lambda}{2 \pi} \tan ^{-1}\left(t_{2}\right), \quad t_{2} \geq 0
\end{aligned}
$$

To analytically find the solution of the open circuit stub impedance, we substitute $t_{1}$ an $t_{2}$ in Equation3.9 to determine the susceptance of the open circuit stub $\left(B_{1}\right.$ and $\left.B_{2}\right)$.

$$
\begin{aligned}
& B_{1}=\frac{x t_{1}^{2}+\left(r^{2}+x^{2}-1\right) t_{1}+x}{R_{S}\left(r^{2}+x^{2}+t_{1}^{2}+2 x t_{1}\right)} \\
& B_{2}=\frac{x t_{2}^{2}+\left(r^{2}+x^{2}-1\right) t_{2}+x}{R_{S}\left(r^{2}+x^{2}+t_{2}^{2}+2 x t_{2}\right)}
\end{aligned}
$$

The electrical lengths in radians of the open circuit stubs, $\mathrm{SO}_{1}$ and $\mathrm{so}_{2}$, can then be found by setting the susceptance of the stubs equal to the opposite of the input susceptance, since we are matching to the complex conjugate of the load impedance. 


$$
\begin{aligned}
& s o_{1}=\frac{-\left(\tan ^{-1}\left(R_{S} B_{1}\right)\right)}{2 \pi} \\
& s o_{2}=\frac{-\left(\tan ^{-1}\left(R_{S} B_{2}\right)\right)}{2 \pi}
\end{aligned}
$$

Given that this process gives two matching solutions, the solution with the shortest transmission lines should be chosen. This will give a more wide band match than longer matching solutions.

Using the process from above, we can now match the complex impedances of the input and output of the diode doubler to each patch antenna, both having input impedance of $50 \Omega$. As shown in Figure 3.4, the input and output impedance of the diode doubler is shown to be $104.5-j 500.6 \Omega$ and $68.85-j 244.7 \Omega$, respectively.

The length of the transmission line and open circuit stub for matching the receive antenna to the diode doubler is calculated to be $28.9 \mathrm{~mm}$ and $37.5 \mathrm{~mm}$, respectively. Similarly, the matching lengths for the transmit antenna to the diode doubler are found to be $13.3 \mathrm{~mm}$ and $22.27 \mathrm{~mm}$, respectively.

\subsection{Simulation Results}

This section will go over the electro-magnetic simulations of the antennas and the final diode doubler were conducted using ADS to accurately describe their behavior. 


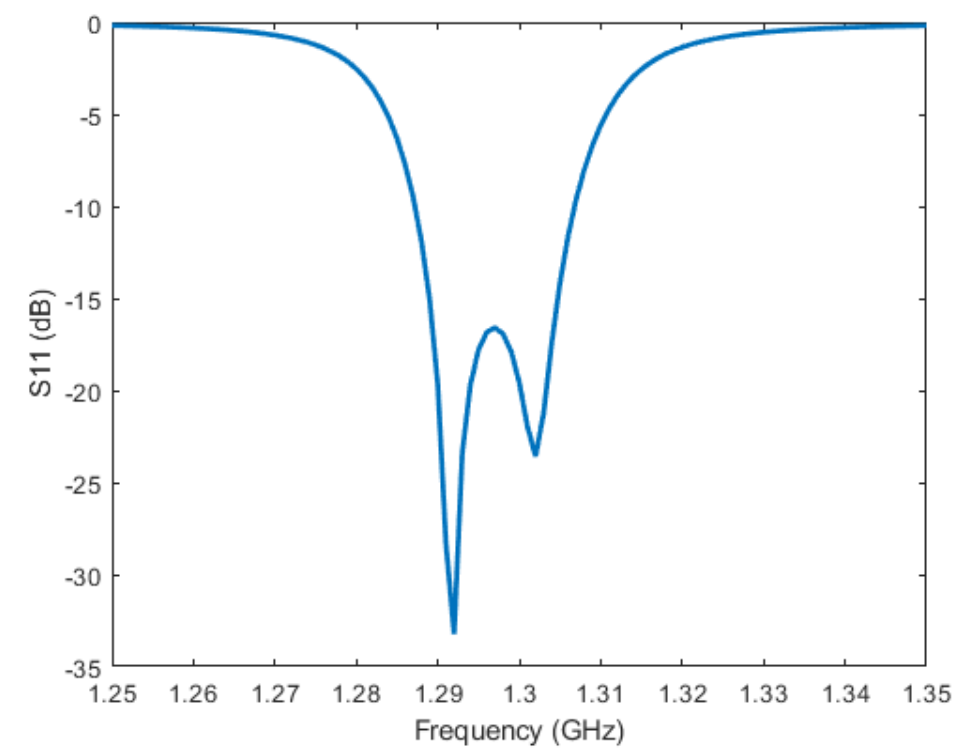

Figure 3.6: Return loss of $1.3 \mathrm{GHz}$ antenna with matching network, relative to diode doubler input impedance. Resonant frequency ranges from 1.29 to $1.31 \mathrm{GHz}$

\subsubsection{Antennas with Matching Networks}

In Section 3.2.2, the process for designing single stub matching scheme for real-tocomplex impedances was introduced. By using the equations in Section 3.2.2, we can plug in the impedance of the antennas (both $50 \Omega$ ) and both the input and output impedances of the diode doubler to calculate the appropriate transmission line lengths to the antennas to the diode doubler. Simulations were conducted using ADS to assess the matching of both antennas to the diode doubler.

The width of the transmission lines can be found simply by knowing the characteristic impedance of the transmission lines, the height of the substrate on which the board is manufactured, and the dielectric constant of the substrate.

To maximize the efficiency of the harmonic transponder, the transmit antenna $(2.6 \mathrm{GHz})$ must radiate at the exact harmonic of the receive antenna. Figures 3.6 


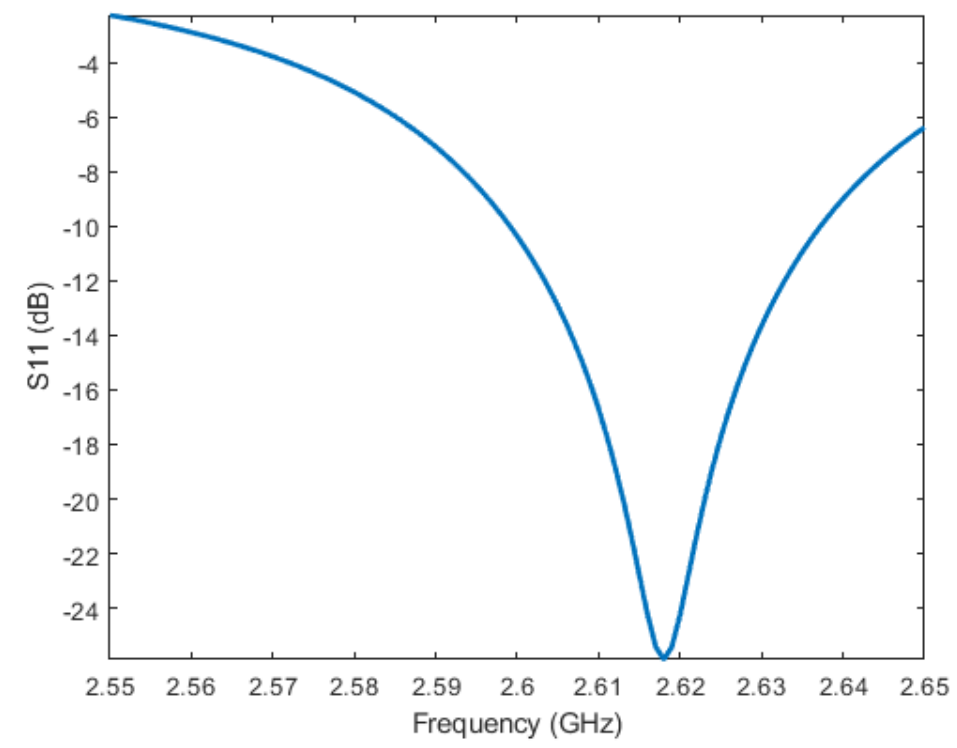

Figure 3.7: Return loss of 2.6 GHz antenna with matching network, relative to diode doubler output impedance. Resonant frequency is found to be $2.619 \mathrm{GHz}$

and 3.7 show the return loss of the $1.3 \mathrm{GHz}$, and $2.6 \mathrm{GHZ}$ antennas, respectively. The receive antenna maintains above $15 \mathrm{~dB}$ return loss for 1.29 to $1.31 \mathrm{GHz}$, while the transmit antenna resonates at around $2.619 \mathrm{GHz}$, indicating that the resonance of these antennas will match perfectly.

\subsubsection{Conversion Loss}

A rather important parameter of a harmonic transponder is its conversion loss. Conversion loss portrays the power difference between the input signal at an intermediate frequency (IF) and a output signal at radio frequency $(\mathrm{RF})$ and is often expressed as a positive number in decibels.

$$
C L=\operatorname{Pin}_{f_{0}}-\text { Pout }_{2 \times f_{0}}
$$




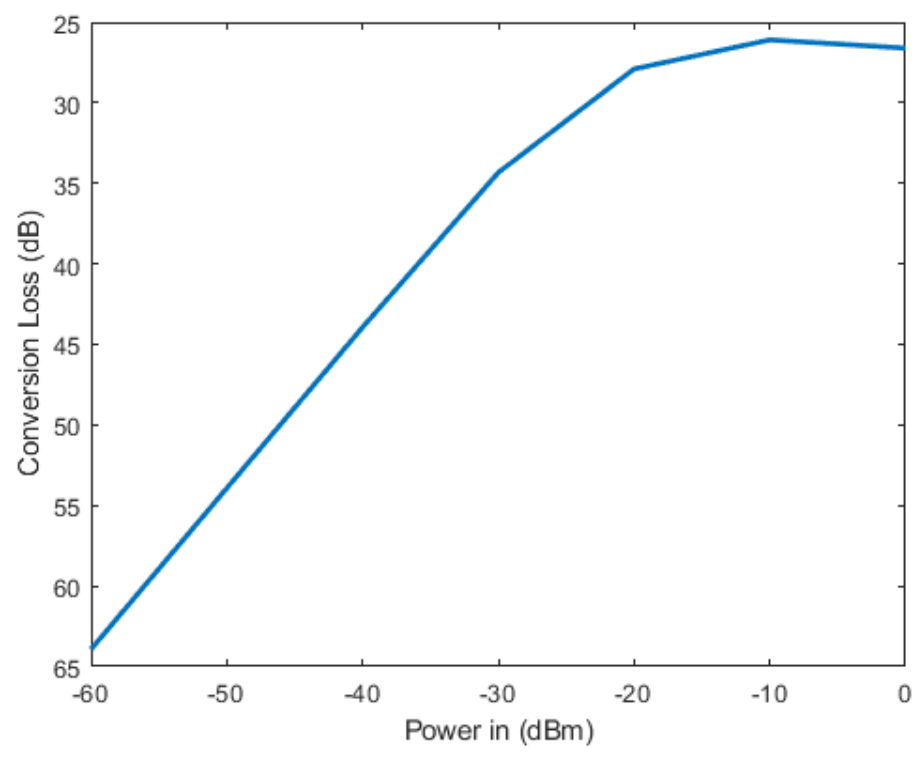

Figure 3.8: Conversion loss of diode doubler with matching circuits connected

The conversion loss simulation included both input and output matching networks. To mimic the patch antennas, each matching network was terminated with a $50 \Omega$ load. Figure 3.8 shows the conversion loss of the diode doubler over a range of input powers. The best conversion loss occurs at $-10 \mathrm{dBm}$, giving a conversion loss of 26 $\mathrm{dB}$. The conversion loss drops off quite fast after an input power of $-20 \mathrm{dBm}$, so it would be advantageous to interrogate no less than -20 dBm at the transponder.

\subsection{Final Harmonic Transponder Layout}

Once matching typologies and patch antennas were designed, a layout was created to use for manufacturing the harmonic transponder, which can be found in Figure 3.9. The cyan colored line represents the edge of the PCB.

The harmonic transponder was manufactured in the High Performance Circuits 


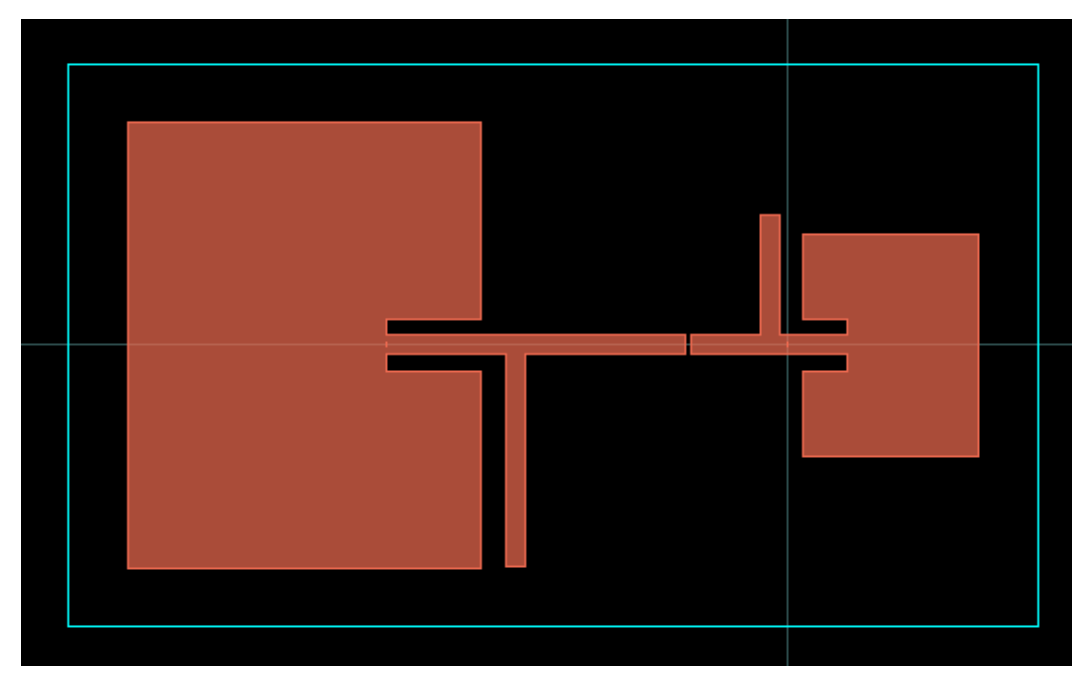

Figure 3.9: Final harmonic transponder design

Laboratory at the University of Vermont (UVM). The antennas and transmission lines were milled onto the Rogers substrate using a PCB milling machine. The main design elements, those being open-circuit transmission line matching and the patch antennas were used since they do not require the need or a top ground plane.

A limitation in the design was not being able to use vias. A via is a conductor that connects two copper layers together through a substrate. The use of vias would have allowed more external matching components to be used, thus making the transponder more compact.

It is also important to notice that the milling machine has an error of $0.3 \mathrm{~mm}$ in position. This could affect the characteristic impedance of the transmission lines. However since the transmission lines used were about $3.2 \mathrm{~mm}$ thick, a $0.3 \mathrm{~mm}$ error will only affect the impedance by about $\pm 3 \Omega$. This impedance variation will have very little affect on the insertion loss of the antennas. Furthermore, the patch antennas are very sensitive to changes in patch size since each patch antenna is designed to resonate at a single frequency. For example, a change in the width of the patch of 
$0.3 \mathrm{~mm}$ could cause an antenna originally designed to resonate at $1.3 \mathrm{GHz}$, to now resonate at $1.25 \mathrm{GHz}$. Seeing as patch antennas are also quite narrow band, this could cause large losses in the harmonic transponder. That being said, this method is very low cost compared to higher precision PCB manufacturing processes.

\subsection{Conclusion}

In this chapter, the design and simulation of a harmonic transponder was presented. Inset feed patch antennas for both the interrogation frequency $(1.293 \mathrm{GHz})$ and the return frequency $(2.586 \mathrm{GHz})$ were designed. To minimize the need for external matching components, a single stub matching scheme was used to match the antennas to the complex impedance of the diode doubler. Conversion loss of the doubler was found to be $26 \mathrm{~dB}$ at an input power of $-10 \mathrm{dBm}$ with the matching elements.

In the next Chapter, we will discuss the software used to interrogate the device presented in this Chapter, and also the FDR from [3]. 


\section{CHAPTER 4}

\section{Software Defined Radio Interroga-}

\section{TOR}

\subsection{FORWARD}

This chapter will discuss preliminary work for developing the SDR interrogator. The work from this section was submitted as is to the WAMICON conference and is still currently in review. The particular harmonic transponder used for this chapter was the frequency doubling reflectenna presented in [3].

\subsection{Interrogator Software}

To the best of the authors' knowledge, interrogation of harmonic transponders have typically utilized a signal generator transmitting at frequency $f_{1}$, with the channel response measured using a spectrum analyzer at frequency $f_{2}=2 \times f_{1}$ [14]. As harmonic transponders are resonant devices, the transmit signal is often swept across 
a band and the response likewise monitored at twice the frequencies. The exception noted, is [43], a complex FPGA-controlled, dual phase lock loop (PLL) system.

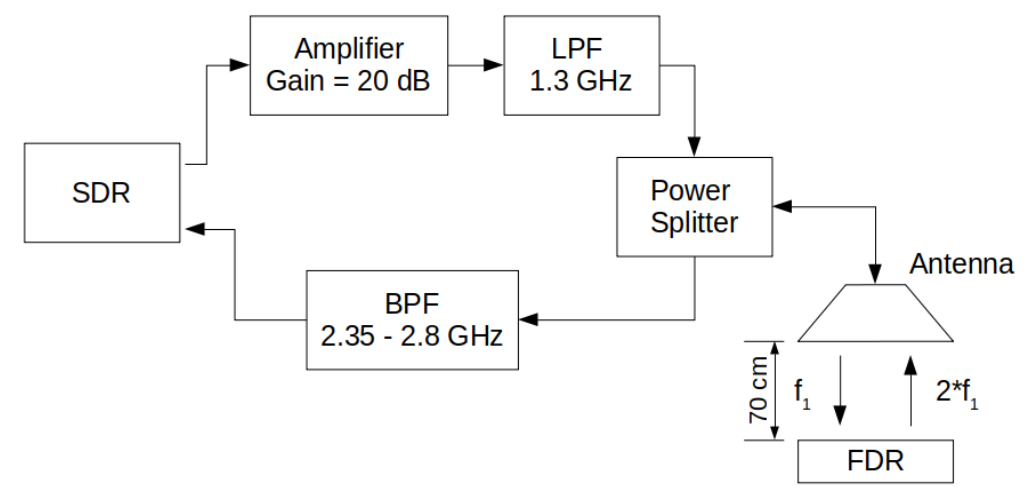

Figure 4.1: Diagram of filtering scheme used for SDR-interrogator

The SDR-based solution presented herein, provides a more flexible, compact and cost-effective alternative. The SDR utilized, the Nuand BladeRF x40, offers a single board solution, eliminating the need for the signal generator and spectrum analyzer combination. The BladeRF x40 costs about $\$ 420$, significantly less than the aforementioned FPGA/PLL solution. The BladeRF is a full-duplex device, meaning it can transmit and receive signals simultaneously. The novelty in this work is that the transmit and receive signals are at two different frequency bands. The receive and transmit ports have separate RF chains allowing each port to be configured to a different frequency, making the BladeRF suitable for the proposed measurement system.

The goal of this work is to excite the FDR over a span of $100 \mathrm{MHz}$ to characterize its behavior. This will produce a response curve that will aid in defining the distance to and the resonant frequency of the FDR.

The interrogation system consists of several off-the-shelf components, such as am- 
plifiers, filters, and power splitters. The antenna used is a Com-Power AH-118-1, a horn antenna with a gain of $10 \mathrm{dBi}$. A photograph of the interrogation system can be found in Fig. 4.3.

The maximum instantaneous bandwidth (IBW) of the BladeRF is $28 \mathrm{MHZ}$, which is not sufficient to view the entire $100 \mathrm{MHz}$ band of interest from the FDR $(2.5 \mathrm{GHz}$ to $2.6 \mathrm{GHz}$ ). However, because the center frequency of the BladeRF is easily adjusted through software, one can readily step the center frequency of both the transmit and receive ports to view the entire $50 \mathrm{MHz}$ (for $f_{1}$ ) and $100 \mathrm{MHz}$ (for $f_{2}$ ) bandwidths. For this work, the IBW of the BladeRF was set to $20 \mathrm{MHz}$.

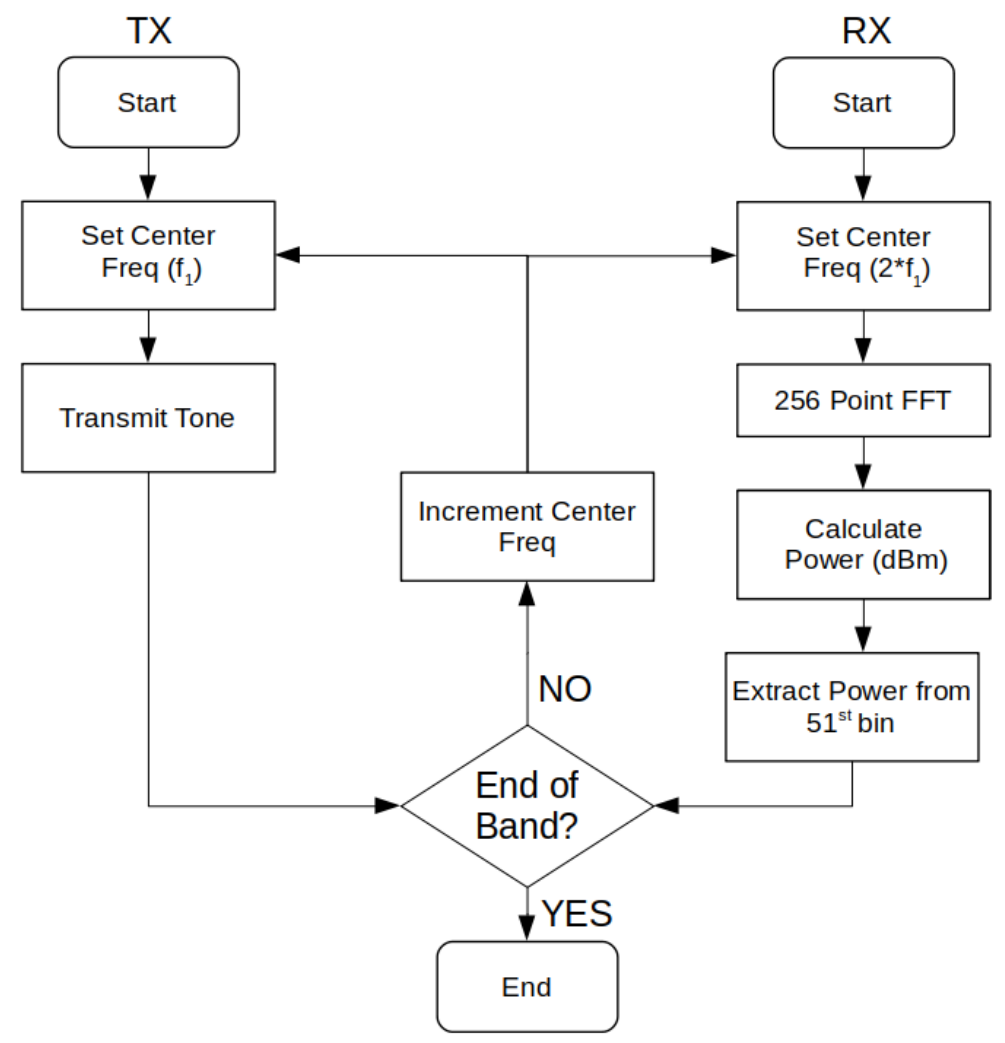

Figure 4.2: Program flowchart of the SDR-based interrogator. "TX" and " $R X$ " are the separate transmit and receive chains of the BladeRF. 


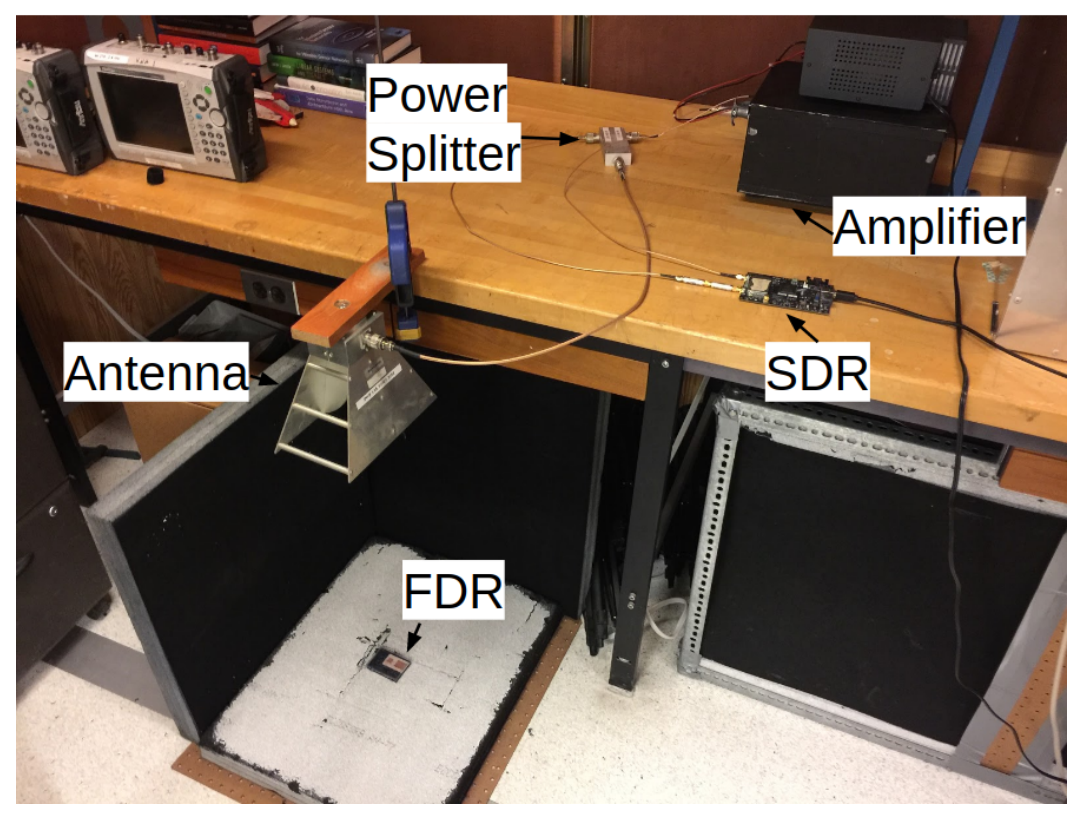

Figure 4.3: SDR interrogator setup. Distance from antenna to FDR is $70 \mathrm{~cm}$. Anechoic foam is used around the FDR during testing to minimize scattering effects.

The transmit signal is a stepped-tone, with $f_{1}$ ranging from $1.25 \mathrm{GHz}(2.5 \mathrm{GHz}$ RX) to $1.3 \mathrm{GHz}(2.6 \mathrm{GHz} \mathrm{RX})$ with a $1 \mathrm{MHz}$ step size. The receive side calculates a 256-point FFT of the incoming IQ data and converts the measurement to $\mathrm{dBm}$ (resolution $\mathrm{BW}=\frac{20}{256} \mathrm{MHZ}=78.125 \mathrm{kHz}$ ). Peak power is extracted from the 25th bin (a bin is a single point of the spectrum from the FFT). The sweep is conducted by modulating a single tone at base-band placed in the center of the 51st bin of the FFT.

Because this SDR-interrogator uses only a single antenna, a power splitter (MiniCircuits ZB2PD-63+) must be used to separate transmit and receive signals. The power splitter provides only some isolation between Ports 1 and 2 (19 dB), so other filters are used to reduce the power of the transmit signal, $f_{1}$. 


\subsection{Results}

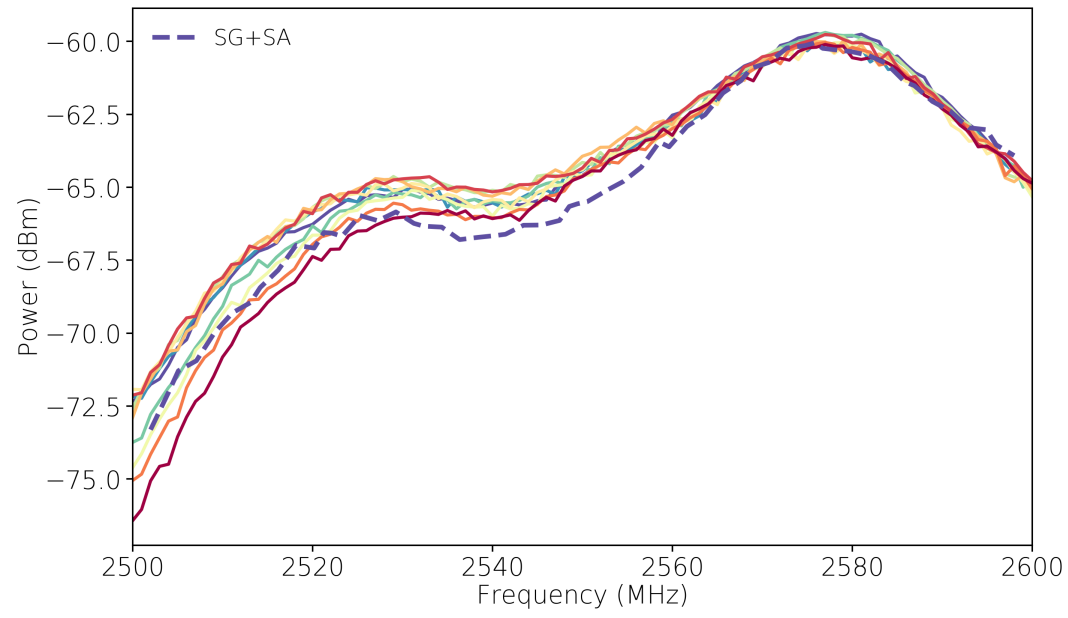

Figure 4.4: SDR vs. signal generator and spectrum analyzer testing of FDR. Transmit EIRP set to $8 \mathrm{dBm}$. Interrogation distance is $70 \mathrm{~cm}$. Peak return frequency is $2.565 \mathrm{GHz}$ (FDR is resonant at 1.2825/2.5650 GHz).

Measurements were taken at $70 \mathrm{~cm}$ with the proposed SDR system, and also with a Agilent E4422B signal generator (SG) and Anritsu MS2063A spectrum analyzer (SA) combination to verify the accuracy of the SDR system. When the BladeRF down-converts received RF energy to base-band, a DC offset in the FFT of the received signal is apparent. To combat this issue, a power measurement is extracted from a single bin about $2 \mathrm{MHz}$ away from the DC bin of the FFT. Fig. 4.4 shows the characteristic curve of the FDR, excited by both the SG+SA combination and the SDR. The mean error between the 10 SDR measurements, and the SG+SA measurements is $1.15 \mathrm{~dB}$, demonstrating that our low-cost SDR system has comparable performance to laboratory grade of measurement equipment. For the intended purpose of detecting and determining the resonant frequencies of FDR-based devices, the author contends this performance will suffice. 
The transmit variable gain amplifier of the BladeRF was incremented over $20 \mathrm{~dB}$ range to observe the minimum detection power of the FDR. The results can be found in Fig. 4.5 where the range was $70 \mathrm{~cm}$, and Fig. 4.6, where several sweeps were taken at a transmit power of $0 \mathrm{dBm}$. We found that with a transmit EIRP of $0 \mathrm{dBm}$ at 70 $\mathrm{cm}$, the peak of the response curve was still $3 \mathrm{~dB}$ above the noise floor by averaging several sweeps to mitigate the effects of the noise.

In practice, there is a desire to interrogate devices over greater distances than illustrated in Fig. 4.3. Based on our measurement, we contend our existing system is capable of interrogating over $70 \mathrm{~cm}$, at $0 \mathrm{dBm}$ EIRP with 5 averages. The current range limitation is due to the external amplifier, which can supply up to $20 \mathrm{~dB}$ of gain. Assuming free space pathloss, an interrogator with a transmit EIRP of $45 \mathrm{dBm}$ could easily detect a FDR about 5 meters from the interrogator.

The SDR interrogator offers several advantages to the SA+SG interrogator. For example, transmit power from the SDR is easily altered by software. The BladeRF, the transmitter gain is programmable to any gain between $-35 \mathrm{~dB}$ to $21 \mathrm{~dB}$ in 1

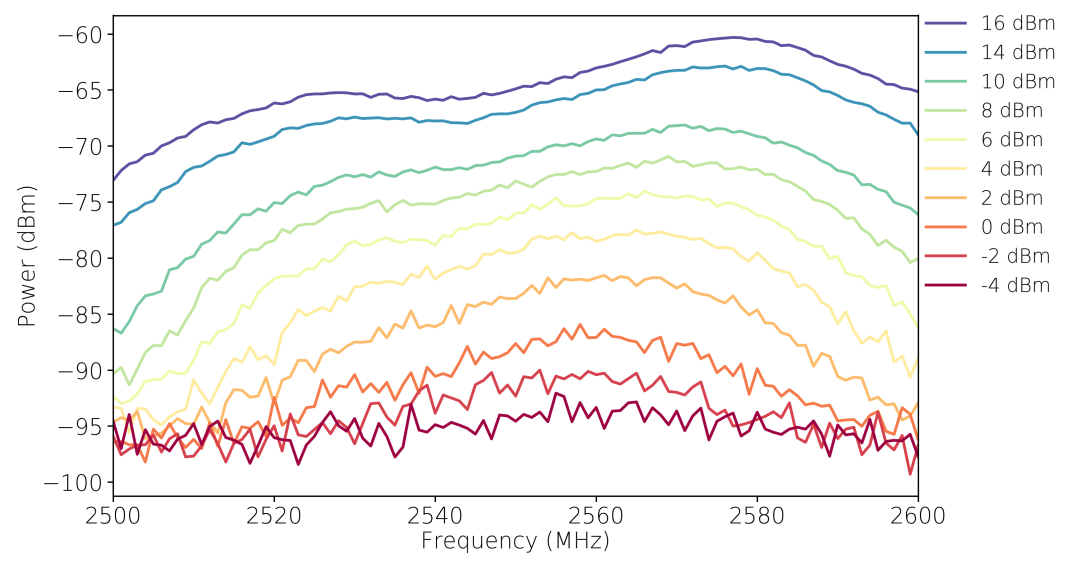

Figure 4.5: Swept transmitted power with associated receive power. Antenna placed $70 \mathrm{~cm}$ away from FDR. 


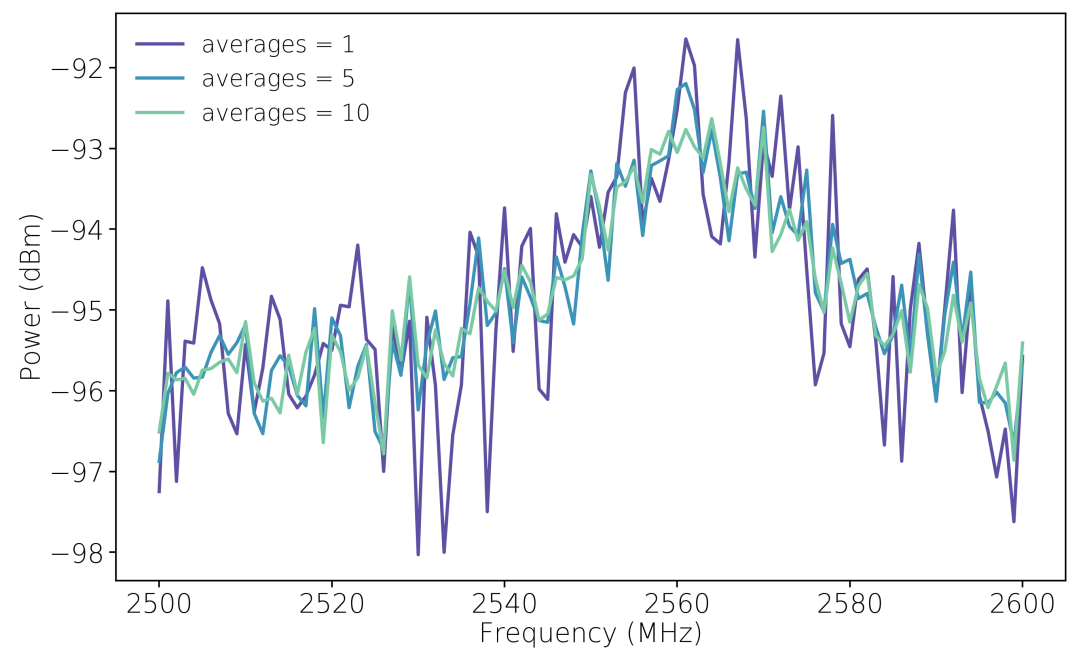

Figure 4.6: TX power set to $0 \mathrm{dBm}$. 5 averages is sufficient for the FDR peak frequency to be $3 \mathrm{~dB}$ above the noise floor.

$\mathrm{dB}$ increments, implying that this interrogator could easily operate over a range of distances. Additionally, the SDR offers high configurability, allowing for rapid prototyping of different interrogation techniques.

Another key advantage that the SDR-interrogator has over the laboratory equipment is quick interrogation time. A single sweep $(2.5 \mathrm{GHz}-2.6 \mathrm{GHz}$ with 78.125 kHz RBW) with the SDR-interrogator takes about 30 seconds, whereas the laboratory equipment took upwards of 5 minutes, since the signal generator and spectrum analyzer are two separate instruments that are operating in two separate bands $(1.25$ to $1.30 \mathrm{GHz}$ and 2.50 to $2.60 \mathrm{GHz}$, respectively). Quick interrogation time would be crucial if this system was mounted on a drone, since flight time is limited. 


\subsection{Conclusion}

In this section, a dual-frequency measurement system capable of interrogating a frequency doubling reflectenna with a single SDR is presented. Mean absolute error was found to be $1.15 \mathrm{~dB}$ when compared to laboratory grade measurement equipment. Interrogation range was measured to be $70 \mathrm{~cm}$ with an interrogation power of $0 \mathrm{dBm}$. This system has demonstrated that it can be comparable to lab equipment while being more compact, quicker, and low cost. 


\section{Chapter 5}

\section{Miniturizing Interrogator And Test-}

\section{ING WITH HARMONIC TRANSONDER}

\subsection{INTRODUCTION}

The previous setup of the harmonic transponder interrogator consisted of several off the shelf components SMA components, including and amplifier, a splitter, and several filters. A smaller interrogator is desired for other applications, such as mounting the interrogator on a drone, or placing the interrogator in a confined space.

Using the BladeRF has proved to be a accurate replacement for the signal generator and spectrum analyzer combination as shown in the previous Chapter. However, the external amplifier and splitter are still both quite large. To minimize the size of the amplifier and splitter, a PCB is designed that is capable of performing the same signal conditioning as the previous setup with a significantly smaller form factor. 


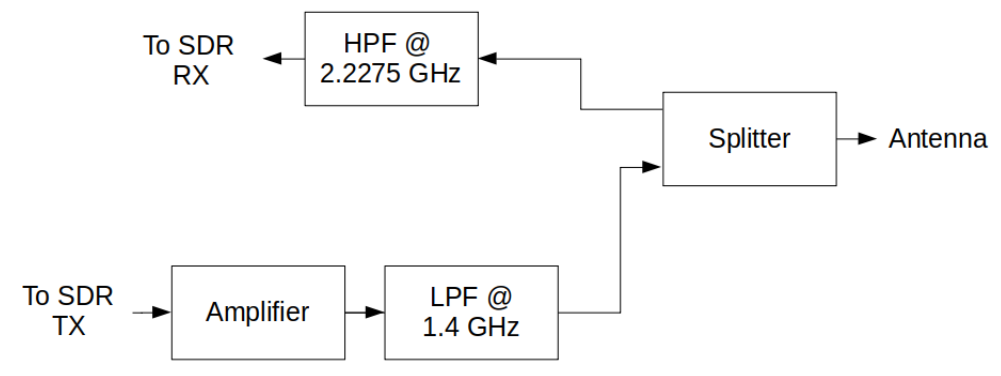

Figure 5.1: Filter and amplifier flow chart

\subsection{Filter AND Amplifier BoARd Design}

This section will go over the design of the PCB filter and amplifier board. The design constraints can be found below, and a flow chart can be found in 5.1:

- Amplification: Amplification of the interrogation signal is necessary to increase to total interrogation range. The interrogator in Chapter 4 used an external amplifier with a gain of about $20 \mathrm{~dB}$, giving a total range for the FDR of around $70 \mathrm{~cm}$ at only $0 \mathrm{dBm}$. In the new interrogator it would also be advantageous to use a similar gain to achieve similar results, thus a MiniCircuits GALI-24+ SMT amplifier is used, which has a gain of $22 \mathrm{~dB}$ at $1 \mathrm{GHz}$.

- Low pass filter (LPF) rejection for the transmit port of the SDR: The BladeRF is a good signal generator but when transmitting towards the maximum of the front-end amplifiers gain, large harmonics can be produced. To combat this issue, low pass filters are used to reject any possible harmonics generated by the SDR. The proposed system uses three LPFs with a cutoff frequency of $1400 \mathrm{MHz}$ to accomplish this task.

- High pass filter (HPF) rejection for the receive port of the SDR: A large 


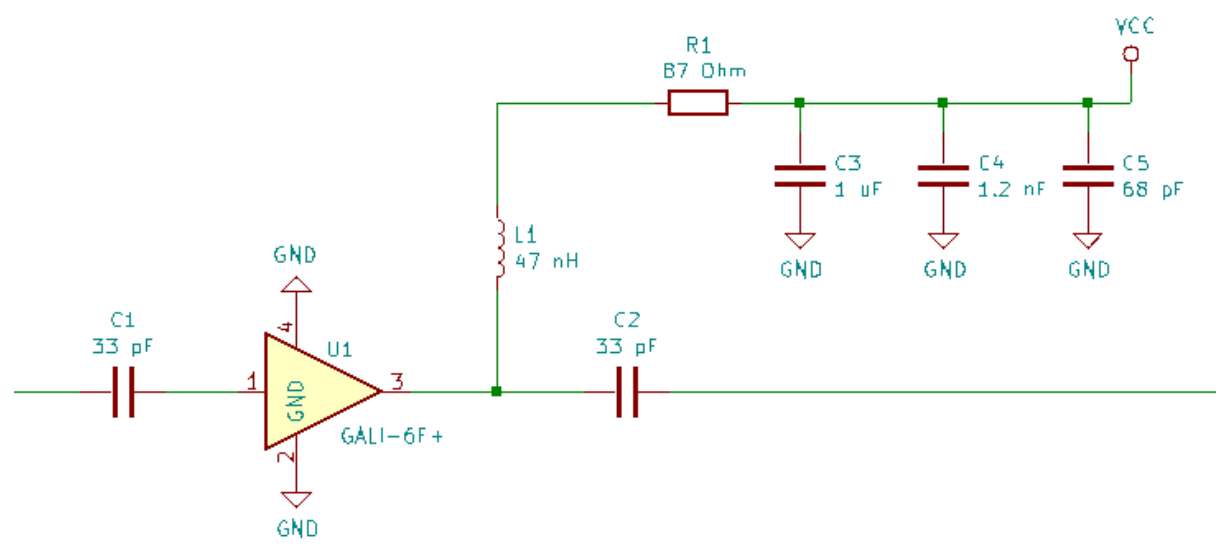

Figure 5.2: Amplifier layout for Minicircuits GALI-29+

portion of the interrogation signal is often still visible in the received signal from the FDR. This is mainly due to the interrogation signal bleeding through from one port to another in the splitter, and reflections of the interrogation signal from the surrounding environment. $2.275 \mathrm{GHz} \mathrm{HPFs}$ are used to remove any of the interrogation signal that could still be present in the received signal.

\subsubsection{Amplifier LAYOUT}

The MiniCircuits GALI-29+ surface mount (SMT) amplifier was used to increase the transmit power of the $1.3 \mathrm{GHz}$ signal coming from the SDR. The circuit layout for this amplifier can be found in Figure 5.2. DC-blocking capacitors are use on the input and output of the amplifier which have a value of $33 \mathrm{pF}$. Bypass capacitors were also used to short any high frequency noise to ground. Three different size capacitors are used to smooth different frequency noise on the PCB. 


\subsubsection{SMT SPLiTTER}

A power splitter was used in the previous interrogator to combine both transmit and receive signals, allowing the use of only a single antenna for interrogation. The surface mount solution to this was the MiniCircuits SEPS-2-33+. This splitter has very similar characteristics to the previous SMA splitter (ZB2PD-63+). The SEPS-2$33+$ provides a frequency range of $600-3000 \mathrm{MHz}$, within the band of interest for the harmonic transponder presented in Chapter 3 and the FDR presented in [3]. It also provides $22 \mathrm{~dB}$ of isolation between Ports 1 and 2, meaning the signals both going to and coming from the antenna will not interfere greatly.

\subsubsection{Filtering}

Removing any unwanted interference is crucial for the operation of a harmonic transponder interrogation system. There are two main points in the system where interference can occur. First, harmonics from the transmitter can interfere with the return signal since they are at the same frequency as stated in Section 5.2. MiniCircuits LFCN1400D LPFs are used to remove any harmonics that could have made it through the amplifier. In addition, two SMA LPFs are used after the transmit port of the SDR to remove any possible harmonics generated by the SDR itself.

For the receive side, and lingering interrogation signal should also be filtered out. A high power interrogation signal could easily saturate the front end of the SDR resulting in faulty measurements. Two MiniCircuits HFCN-2275+ SMT HPFs are used to midigate this issue. 


\subsubsection{Final PCB LAYOUT}

To minimize variation in the microstrip trace characteristic impedance, soldermask was removed from all traces. Since solder mask has a certain dielectric constant, adding it on top of a transmission line can have an effect on the transmission line's characteristic impedance. The width of the microstrip traces is dependent on a few factors, those being the height of the substrate, and the dielectric constant of the substrate.

$$
\begin{gathered}
\epsilon_{e f f}=\frac{\epsilon_{R}+1}{2}+\frac{\epsilon_{R}-1}{2 \sqrt{1+12 \frac{H}{W}}} \\
Z_{0}=\frac{120 \pi}{\sqrt{\epsilon_{\text {eff }}}\left[\frac{W}{H}+1.393+\frac{2}{3} \ln \left(\frac{W}{H}+1.444\right)\right.}
\end{gathered}
$$

All components were connected with $50 \Omega$ transmission lines to minimize losses between components. The characteristic impedance of a microstrip transmission line can be calculated using the equations above, as long as the width of the transmission line is longer than height of the substrate. The substrate used was 30 mil FR4 from OSHPARK, which has a dielectric constant of 4.5 [44]. Using equations 5.1 and 5.2, we find that a track width of 55 mil results in a $50 \Omega$ characteristic impedance. Final PCB layout can be found in Figure 5.3.

\subsection{Measurement Results}

To validate the filtering and amplifying characteristics of the board from Section 5.2, scattering parameters were examined with a Anritsu VNA. 


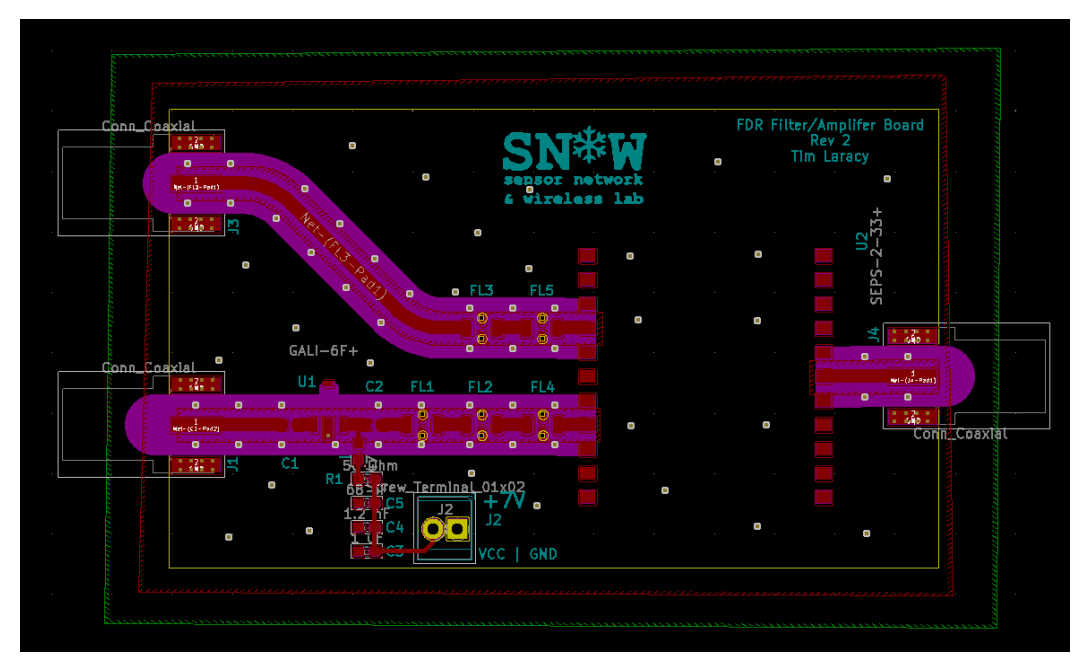

Figure 5.3: Filter and amplifier board layout

\subsubsection{RETURN LOSS}

Observing Figure 5.4, it is clear that there are some matching issues with the board. The return loss of the TX path hovers around $6 \mathrm{~dB}$, meaning only about $75 \%$ of the input power is going to the board, while the other $25 \%$ is being reflected. A good return loss value is considered to be $15 \mathrm{~dB}$, where only $3 \%$ of the input power is reflected. There are a few reason the reflections on the TX path are so high. One, the incorrect transmission line width could have been used, however this is not the case since the transmission line values were calculated using ADS. Two, there could be a grounding issue. It is quite difficult to heat the large ground place on the top of the board since the point of contact of a typical soldering iron is so small. The ground leads on the connectors were very hard to heat to a full capacity, which lead to something called a "cold joint", where the solder does not fully adhere to the board material or the part.

In manufacturing settings, hotplates are often used to heat the entire ground plane 


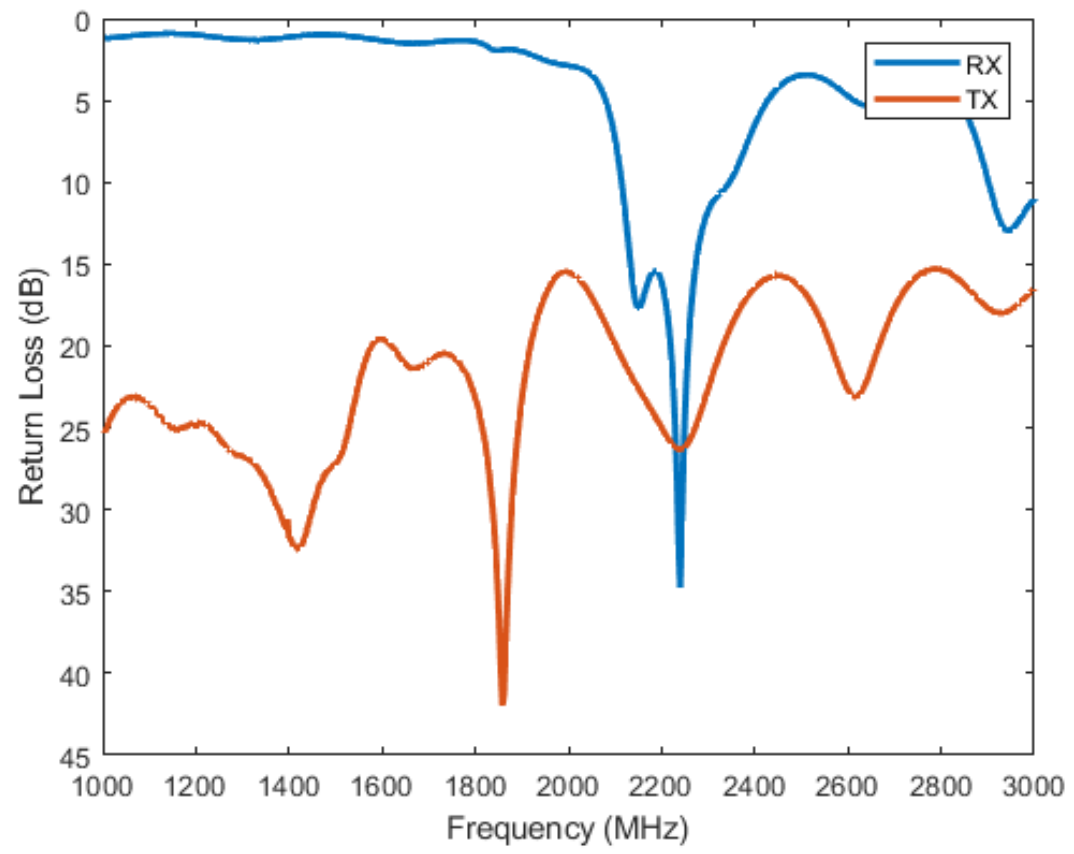

Figure 5.4: S11 of both RX and TX Path (ports on left side of Figure 5.3

making it much faster to heat up when solder, in hindsight this could have been very effective when soldering this board.

\subsubsection{INSERTION LOSS}

The transmit path is responsible for two things: amplifying the interrogation signal, and eliminating any harmonics that could be generated by the amplifier. The insertion loss of this part of the board can be found in Figure 5.5.

Ideally, the gain of this circuit would peak at around $20 \mathrm{~dB}$ due to the gain of the amplifier. A large amount rejection would also be seen due to the LPFs placed after the amplifier. This path maintains about $14.4 \mathrm{~dB}$ of gain with $57.6 \mathrm{~dB}$ of rejection at $2600 \mathrm{MHz}$. 


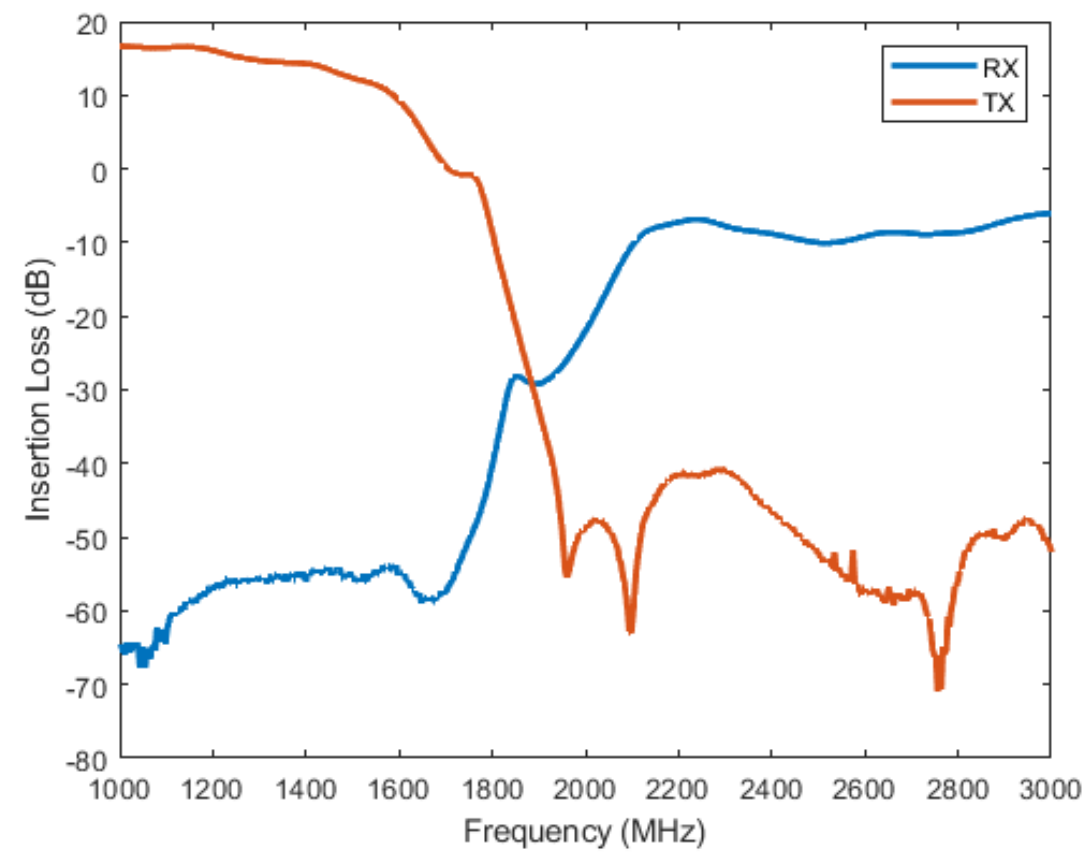

Figure 5.5: S21 response of the both $R X$ and $T X$ path

The receive path also had some losses likely due to a poor soldering joint. Filtering characteristics are good at $1.3 \mathrm{GHz}$, resulting with about $55.7 \mathrm{~dB}$ of rejection. Insertion loss at $2.6 \mathrm{GHz}$ is lower than anticipated, with $7.4 \mathrm{~dB}$ at $2.6 \mathrm{GHz}$.

\subsection{INTERROGATION}

The TX and RX ports of the SDR were connected to the filter and amplifier board designed in the previous section using SMA cables. The antenna port (the port on the right side in Figure 5.3) was then connected to a Com-Power AH-118-1 antenna. There were some issues with extra harmonics being generated by the SDR, so two extra SMA LPFs with a cutoff frequency of $1.4 \mathrm{GHz}$ were connected to the TX port of the SDR as well. 


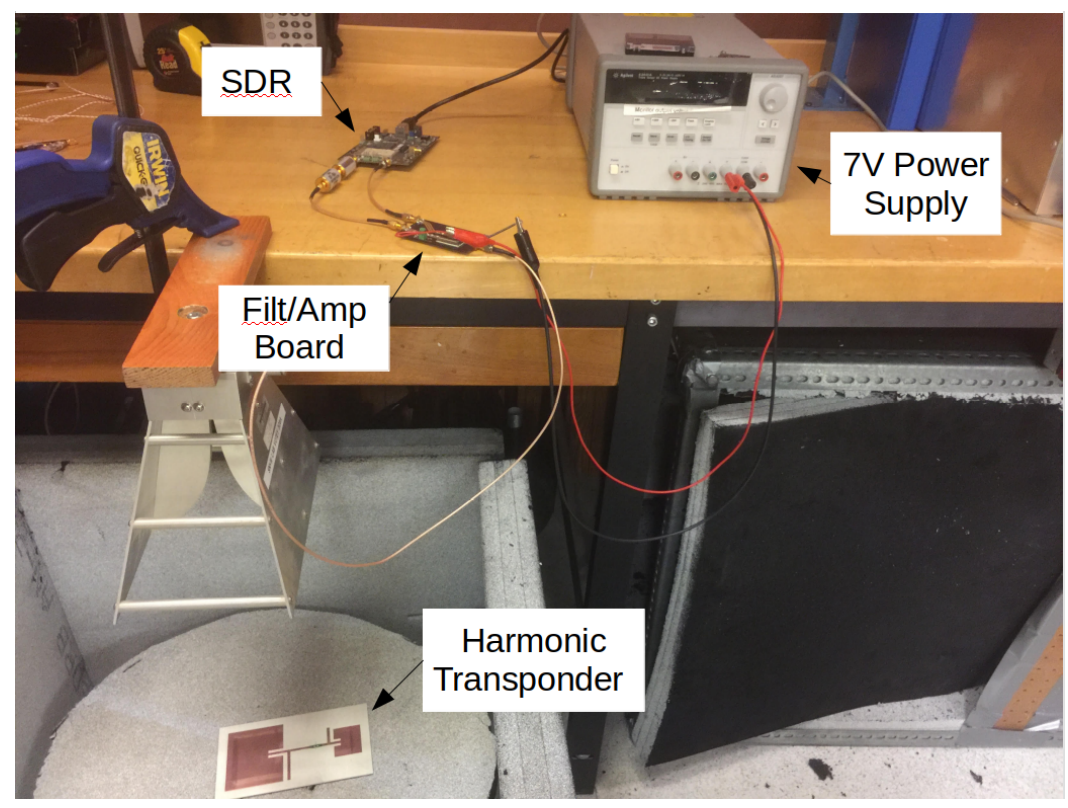

Figure 5.6: Final setup for interrogating harmonic transponder

The harmonic transponder designed in Chapter 3 was placed $53.3 \mathrm{~cm}$ away from the antenna, and surrounded by anechoic foam to reduce interference in the surrounding laboratory environment.

A 7V supply was used to power the filter and amplifier board. When the setup was interrogating, the power supply was drawing $30 \mathrm{~mA}$. The final interrogation setup can be found in Figure 5.6. A laptop computer was used to both power and access the SDR via a USB port.

Interrogation software similar to that presented in Chapter 4 was used for data collection. There are several amplifiers on the front end of the BladeRF for both transmitting and receiving. The receiver has two integrated low-noise amplifiers that can easily be programmed through software. These amplifiers were modified to mitigate some of the loss issues in the PCB. The front-end RX LNA was set to $15 \mathrm{~dB}$ which accounted for the losses in the RX side of the filter and amplifier PCB. 


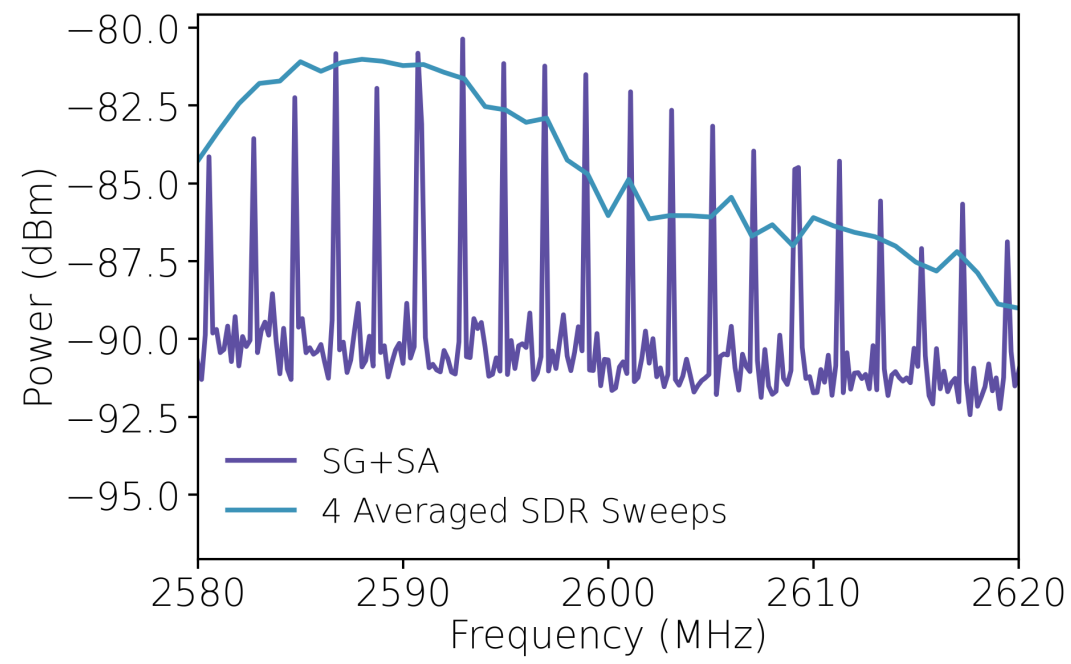

Figure 5.7: Harmonic transponder at a distance of $53.3 \mathrm{~cm}$ from the antenna. $S G+S A$ setup is the same as used in Chapter 4. The EIRP of the setup is $5 \mathrm{dBm}$

The final result can be found in Figure 5.7, which was compared to the signal generator and spectrum analyzer combination mentioned in Chapter 4. Mean error between the peaks of the $\mathrm{SG}+\mathrm{SA}$ and the averaged SDR sweeps is $2.17 \mathrm{~dB}$. This shows there there is some distortion introduced by the filter and amplifier board compared to the results from Chapter 4, where the mean error was $1.15 \mathrm{~dB}$.

\subsubsection{Calculated Transponder Metrics}

To assess the performance of the harmonic transponder, two metrics were calculated. Those being the total conversion loss, and the $10 \mathrm{~dB}$ bandwidth.

\section{Conversion Loss}

As explained in section 3.3.2, conversion loss is one of the most important metrics for harmonic transponders. To estimate the actual conversion loss of the harmonic transponder, a link budget can be used to account for other losses or gains in the 


\begin{tabular}{|c|c|}
\hline Transmitted Power (dBm) & -5 \\
\hline Transmit @ 1.3 GHz Antenna Gain (dBi) & 9 \\
\hline Forward Link Path Loss (dB) & 29.21 \\
\hline Return Link Path Loss (dB) & 35.23 \\
\hline Receive @ 2.6 GHz Antenna Gain (dBi) & 11 \\
\hline Received Power (dBm) & -80.49 \\
\hline Conversion Loss (dB) & $\mathbf{3 1 . 0 4}$ \\
\hline
\end{tabular}

Table 5.1: Link budget for calculating the conversion loss of the harmonic transponder

system, such as path loss, antenna gains, antenna power, etc. A typical link budget in free space can be found in the equation below:

$$
P_{R X}=P_{T X}+G_{T X}+G_{R X}-L_{F S}
$$

Where $P_{R X}$ is received power $(\mathrm{dBm}), P_{T X}$ is transmitter output power $(\mathrm{dBm}), G_{T X}$ is transmitter antenna gain $(\mathrm{dB}), G_{R X}$ is receiver antenna gain $(\mathrm{dB})$, and $L_{F S}$ is the free space path loss $(\mathrm{dB})$, which can be given by the following equation:

$$
F S P L=20 \log _{10}\left(\frac{4 \pi d f}{c}\right)
$$

To evaluate the conversion loss of the harmonic transponder, the link budget changes slightly. Path loss at both interrogation and return return signals. The median peak power of the sweeps occurred at $2.599 \mathrm{GHz}(1.2995 \mathrm{GHz}$ interrogation frequency). This is the frequency that will be used to calculate total conversion loss of the harmonic transponder. The link budget for the harmonic transponder can be found in the equation below: 


$$
P_{R X}=P_{T X}+G_{T X}+G_{R X}-L_{I N T F S}-L_{R E T F S}-L_{c o n v}
$$

Where $L_{I N T F S}$ and $L_{R E T F S}$ are the free space path loss values for the interrogation and return frequencies, respectively. The conversion loss can easily be solved for knowing the other values. Using equation 5.5, which is illustrated in Table 5.1, we find the total conversion loss of the harmonic transponder to be $31.04 \mathrm{~dB}$ at an input power of $-28.21 \mathrm{dBm}$.

\section{$10 \mathrm{~dB}$ Bandwidth}

For sensing applications, it would be advantageous to know what the bandwidth this device is. The $10 \mathrm{~dB}$ bandwidth was calculated for the median of all four signals seen in Figure 5.7. The two $-10 \mathrm{~dB}$ points from the peak at $2.599 \mathrm{GHz}$ were found to be $2.572 \mathrm{GHz}$ and $2.624 \mathrm{GHz}$. This results in a $10 \mathrm{~dB}$ bandwidth of $52 \mathrm{MHz}$. This metric could be important if a sensing medium were added to the harmonic transponder. The sensing medium could vary the bandwidth of the device, or shift the peak depending on an LC resonant circuit.

\subsubsection{Conclusion}

Miniaturization of the SDR interrogator was presented. A PCB was developed to decrease the size of the interrogator while still maintaining good signal conditioning characteristics. The $1.3 \mathrm{GHz}$ interrogation signal was shown to be amplified by 14.4 $\mathrm{dB}$ while still maintaining good filtering characteristics, rejecting the return signal at 2.6 $\mathrm{GHz}$ by $57.6 \mathrm{~dB}$. The $2.6 \mathrm{GHz}$ portion of the board also showed good filtering 
characteristics, resulting with $55.7 \mathrm{~dB}$ of rejection at $1.3 \mathrm{GHz}$.

Additionally, the full interrogation system was tested in this chapter to verify its performance. The Fharmonic transponder presented in Chapter 3 was shown to be successfully interrogated at no more than $53.3 \mathrm{~cm}$ away with an interrogation power of $5 \mathrm{dBm}$. Conversion loss of the transponder was shown to be $31.04 \mathrm{~dB}$ with -28.21 $\mathrm{dBm}$ input power at the device. 


\section{CHAPTER 6}

\section{Conclusions And Future Work}

\subsection{Contributions}

In this thesis, a software defined radio interrogator for harmonic transponders is presented. Compared to interrogators from prior literature, this system is much more compact. This interrogator is capable of interrogating two different harmonic transponder devices over the frequency range of 2.50 - 2.65 GHz, with accuracy comparable to laboratory instrumentation. A harmonic transponder was also designed using microstrip transmission line elements and single Schottkey diode.

\section{- Design of a transmission line matched harmonic transponder}

A harmonic transponder was designed to test the entire system. This transponder used two typical patch antennas for transmit and receive, each tuned to their respective frequency. Using traditional design methods, the input impedance of each antenna was calculated to be $50 \Omega$ at the input. To match the real impedance of the antennas to the complex load impedance of the diode doubler, a single stub 
transmission line matching method was used. This eliminates the need for any external matching components, making it much simpler and more robust.

- Development of algorithm for interrogating a harmonic transponder using SDR

Chapter 4 covers the design of an algorithms for interrogating and receiving signals from a harmonic transponder. This system was then used in Chapter 5 to interrogate the harmonic transponder presented in Chapter 3. To combat the non uniform noise figure of the BladeRF, only the center frequency was modified to change the frequency of interrogation. A tone was placed in the 50th bin of the received signals FFT, which was extracted at each center frequency. Mean error was shown to be $1.15 \mathrm{~dB}$ when compared to laboratory instrumentation.

Additionally, a compact filter and amplifier board were designed, minimizing the entire system drastically.

- Demonstration of the entire system To finalize the work of this thesis, the entire harmonic transponder system was tested. The harmonic transponder from Chapter 3 was interrogated at a distance of $32 \mathrm{~cm}$ from the interrogation antenna at a transmit power of $10 \mathrm{dBm}$ EIRP. The characteristic curve of the tag was then used to calculate the $10 \mathrm{~dB}$ bandwidth of the device, which will be crucial for knowing if sensing ability was then added to the tag. $10 \mathrm{~dB}$ bandwidth was found to $50 \mathrm{MHz}$. 


\subsection{Future Work - Interdigital CAPAC- ITOR}

Some harmonic transponder devices mentioned in Chapter 2 were shown to have an embedded sensing ability, even without external power. The author proposes the idea of using an interdigital capacitor etched into a PCB to add sensing ability to the harmonic transponder.

Interdigital capacitors are interwoven microstrip fingers spaced apart by a certain distance. The dielectric for an interdigital capacitor then becomes the air of above the microstrip. Any change in dielectric constant above the interdigital capacitor will then correspond to a change in capacitance, the following equation is for a parallel plate capacitor, but the concept is the same for interdigital capacitors:

$$
C=\frac{\epsilon A}{d}
$$

Interdigital capacitors have been shown to be very effective for soil moisture applications $[45,46]$. By placing soil over the fringing field of a interdigital capacitor, the dielectric of the capacitor becomes the soil. Adding moisture to the soil essentially increases the dielectric constant of the capacitor, in turn increasing the capacitance. Figure 6.1 shows a capacitance varying from $0.5-2 \mathrm{pF}$ placed after the diode doubler in the harmonic transponder designed in Chapter 3.

Figure 6.2 shows a possible layout for incorporating an interdigital capacitor into a harmonic transponder. 


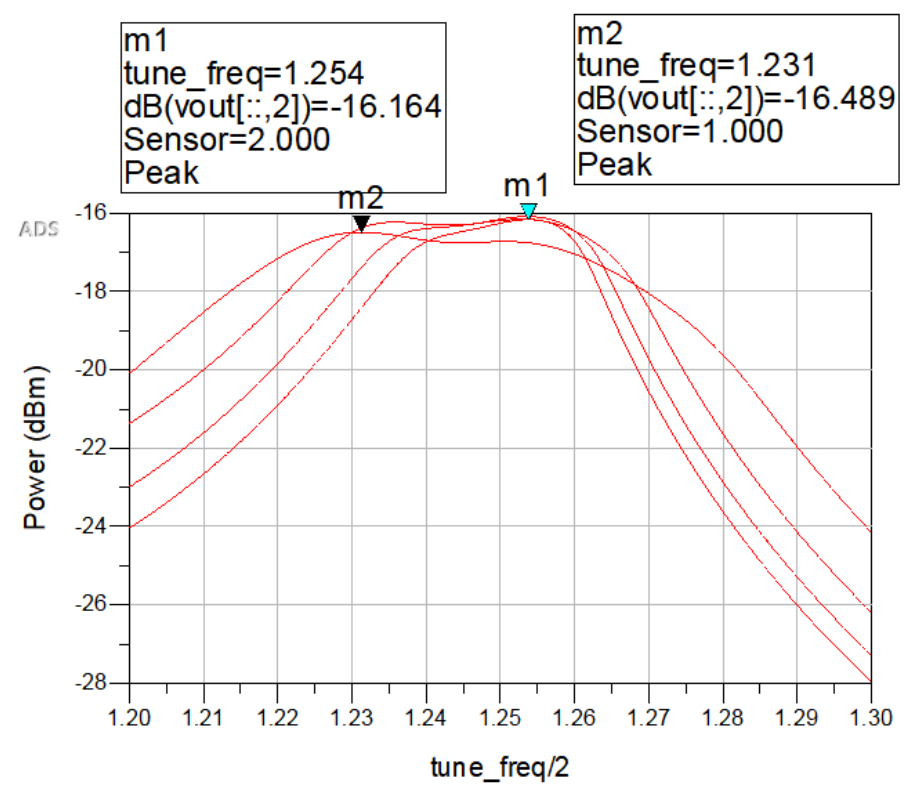

Figure 6.1: Simulated capacitance varied from 0.5-2 pF. Resultant shift in peak is $23 \mathrm{MHz}$.

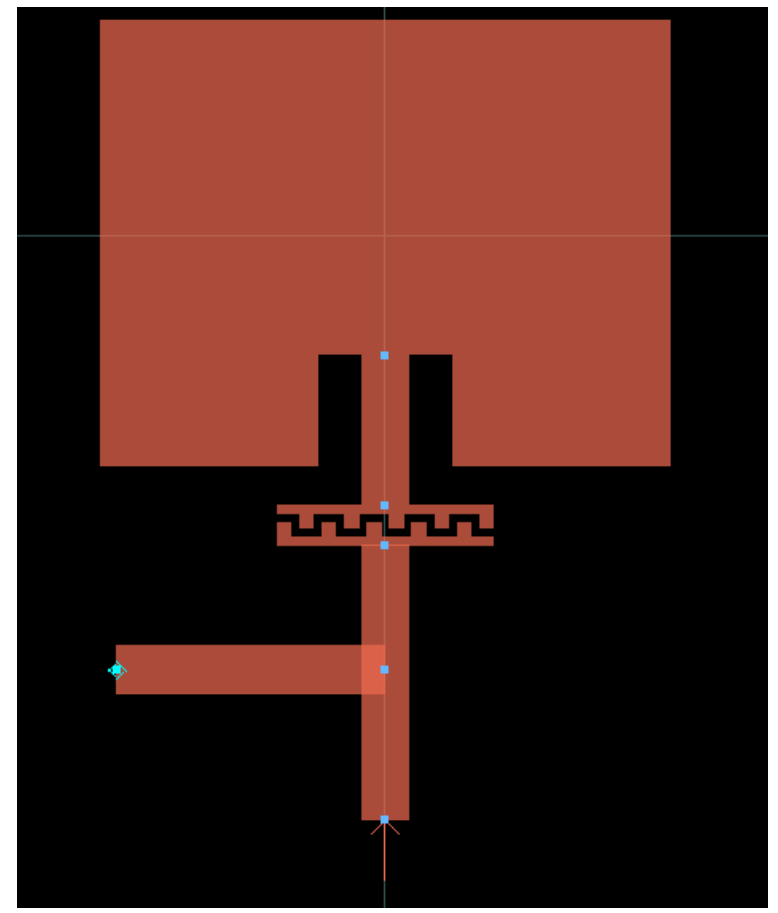

Figure 6.2: 2.6 GHz antenna layout with interdigital capacitor integrated. 


\subsection{FINAL WORDS}

Low cost hardware and open source tools have enabled many engineers, scientists and hobbyists alike to access the RF spectrum like never before. In this work, we have presented a low cost, dual-frequency measurement system capable of interrogating harmonic transponders with a single SDR. Mean absolute error was found to be 1.15 $\mathrm{dB}$ when compared to laboratory grade measurement equipment. Interrogation range was measured to be $70 \mathrm{~cm}$ with an interrogation power of $0 \mathrm{dBm}$. This system has demonstrated that it can be comparable to lab equipment while being more compact, quicker, and low cost. The size of the interrogator was also minimized leveraging lowcost PCB manufacturing services. Commercial off the shelf components were used to develop a PCB with similar characteristics to the system demonstrated in Chapter 4.

Additionally, the design and simulation of a harmonic transponder was presented. This harmonic transponder was able to be manufactured on a PCB milling machine. Though there were extra losses due to the manufacturing process, measured conversion gain of the transponder was found to be $-31.04 \mathrm{~dB}$ at $-28.21 \mathrm{dBm}$ interrogation power at the tag.

This thesis explored several low-cost options to develop a fully operating harmonic transponder system. This idea could be expanded by exploring applications of this system, which could involve adding a sensing element or more deeply comparing harmonic transponders to other passive sensing systems. 


\section{BIBLIOGRAPHY}

[1] How RFID Works \& Antenna Design: EAGLE: Blog, Oct 2017.

[2] B. G. Colpitts and G. Boiteau. Harmonic radar transceiver design: miniature tags for insect tracking. IEEE Transactions on Antennas and Propagation, 52(11):2825-2832, 2004.

[3] S. M. Presas, T. M. Weller, S. Silverman, and M. Rakijas. High efficiency diode doubler with conjugate- matched antennas. In 2007 European Microwave Conference, pages 250-253, Oct 2007.

[4] José María Núñez Ortuño and Carlos Mascareñas y. Pérez-Iñigo. Software defined radio (SDR) on radiocommunications teaching. 2016.

[5] W. C. Wilson, D. F. Perey, G. M. Atkinson, and R. O. Barclay. Passive wireless saw sensors for ivhm. In 2008 IEEE International Frequency Control Symposium, pages $273-277,2008$.

[6] S. G. Allison S. L. DeHaven A. Ghoshal W. H. Prosser, M. C. Wu. "structural health monitoring sensor development at nasa langley research center. 092020.

[7] W. C. Wilson and G. M. Atkinson. Passive wireless sensor applications for nasaâs extreme aeronautical environments. IEEE Sensors Journal, 14(11):3745-3753, 2014.

[8] Wireless Surface Acoustic Wave (SAW) Sensor Arrays, Oct 2013.

[9] Patrick W Fink, Gregory Y Lin, Timothy F Kennedy, and Phong H Ngo. Systems and methods for RFID-enabled pressure sensing apparatus, Apr 2015.

[10] Ichiro Yoshida. Automatic toll collection system for automotive vehicle, December 2 2003. US Patent 6,658,392.

[11] Geof Wheelwright. Tech on the slopes: How rfid is changing the experience of skiing and snowboarding, Apr 2017. 
[12] Recent Developments in Miniaturized Planar Harmonic Radar Antennas. Zenodo, November 2008.

[13] B. Kubina, C. Mandel, M. Sch $\breve{A} z \breve{A l e r, ~ a n d ~ R . ~ J a k o b y . ~ C o m p a c t ~ q u a s i-c h i p l e s s ~}$ harmonic radar sensor with a dielectric resonator antenna. In 2015 IEEE MTT-S International Microwave Symposium, pages 1-3, 2015.

[14] J. Frolik, J. E. Lens, M. M. Dewoolkar, and T. M. Weller. Effects of soil characteristics on passive wireless sensor interrogation. IEEE Sensors Journal, 18(8):34543460, April 2018.

[15] Nuand BladeRF-x40. https://www.nuand.com/product/bladerf-x40/.

[16] H. M. Aumann and N. W. Emanetoglu. A wideband harmonic radar for tracking small wood frogs. In 2014 IEEE Radar Conference, 2014.

[17] S. Bottigliero, D. Milanesio, M. Saccani, R. Maggiora, A. Viscardi, and M. M. Gallesi. An innovative harmonic radar prototype for miniaturized lightweight passive tags tracking. In 2019 IEEE Radar Conference (RadarConf), pages 1-6, April 2019.

[18] H. Huang, P. Chen, C. Hung, R. Gharpurey, and D. Akinwande. Frequency hopped wireless passive sensing system with harmonic transponder antenna sensor. In 2015 IEEE MTT-S International Microwave Symposium, pages 1-4, May 2015.

[19] About GNU Radio. https://www.gnuradio.org/about/. Accessed: 2019-11-09.

[20] Jennifer Landt. The history of RFID. IEEE Potentials, 24:8-11, 2005.

[21] R. Want. An introduction to RFID technology. IEEE Pervasive Computing, $5(1): 25-33,2006$.

[22] R. Want. Enabling ubiquitous sensing with RFID. Computer, 37(4):84-86, 2004.

[23] M. Roberti. What Are the Typical RFID Tag Frequencies? https://www.rfidjournal.com/blogs/experts/entry?5134. Accessed: 2019-12-01.

[24] Y. Ma and E. C. Kan. Multi-path interference reduction in passive NLTL RFID tags. In 2013 IEEE International Wireless Symposium (IWS), pages 1-4, April 2013.

[25] M. I. M. Ghazali, S. Karuppuswami, and P. Chahal. 3-D Printed Embedded Passive Harmonic Sensor Tag as Markers for Buried Assets Localization. IEEE Sensors Letters, 3(4):1-4, April 2019. 
[26] RECCO Technology. https://recco.com/technology/.

[27] DANIEL MASCANZONI and HENRIK WALLIN. The harmonic radar: a new method of tracing insects in the field. Ecological Entomology, 11(4):387-390, 1986.

[28] N. Tahir and G. Brooker. The investigation of millimetre wave optical harmonic transponders and radar for monitoring small insects. In 2013 IEEE Topical Conference on Wireless Sensors and Sensor Networks (WiSNet), pages 22-24, Jan 2013.

[29] D. Psychoudakis, W. Moulder, C. Chen, H. Zhu, and J. L. Volakis. A portable low-power harmonic radar system and conformal tag for insect tracking. IEEE Antennas and Wireless Propagation Letters, 7:444-447, 2008.

[30] S. Karuppuswami, A. Kaur, M. I. M. Ghazali, and P. Chahal. RFID compatible sensor tags for remote liquid sample interrogation. In 2016 IEEE 66th Electronic Components and Technology Conference (ECTC), pages 2401-2407, May 2016.

[31] The Mathematical Prediction of Radio Waves - 1873. http://www.sparkmuseum.com/BOOK_MAXWELL.HTM.

[32] How to Demodulate an AM Waveform https://www.allaboutcircuits.com/textbook/radio-frequency-analysisdesign/radio-frequency-demodulation/how-to-demodulate-an-am-waveform/.

[33] AD9361. https://www.analog.com/en/products/ad9361.html.

[34] Dimitri Stolnikov. GR-Osmocom. https://git.osmocom.org/gr-osmosdr/, 2014.

[35] Understanding ffts and windowing. https://download.ni.com/evaluation/pxi/Understanding\%2

[36] K. Bansal D. Bhalla. Design of a rectangular microstrip patch antenna using inset feed technique. In IOSR Journal of Electronics and Communication Engineering, volume 7, pages 8-13, 2013.

[37] RO4350B Laminates - Rogers Corporation. https://rogerscorp.com/advancedconnectivity-solutions/ro4000-series-laminates/ro4350b-laminates.

[38] D. Orban and G.J.K. Moernaut. The basics of patch antennas, updated. In Orban Microwave Products, 2009.

[39] SMS Series Schottky Diode . https://www.skyworksinc.com/products/diodes/sms7630series. 
[40] K. Rasilainen, J. Ilvonen, and V. Viikari. Antenna matching at harmonic frequencies to complex load impedance. IEEE Antennas and Wireless Propagation Letters, 14:535-538, 2015.

[41] K. Rasilainen, J. Ilvonen, J. Hannula, and V. Viikari. Designing harmonic transponders using lumped-component matching circuits. IEEE Antennas and Wireless Propagation Letters, 16:246-249, 2017.

[42] Ali A. Behagi. RF and microwave circuit design: a design approach using (ADS). Techno Search, 2015.

[43] V. Sakamuri and J. Frolik. Design of a $2.4 \mathrm{GHz}$ interrogator for a rectennabased, wireless sensor system. In 2011 IEEE Wireless and Microwave Technology Conference, April 2011.

[44] Oshpark 2 layer 2oz $0.8 \mathrm{~mm}$ service. https://docs.oshpark.com/services/twolayer-hhdc/.

[45] M. Protim Goswami, B. Montazer, and U. Sarma. Design and characterization of a fringing field capacitive soil moisture sensor. IEEE Transactions on Instrumentation and Measurement, 68(3):913-922, 2019.

[46] N. S. S. M. da Fonseca, R. C. S. Freire, A. Batista, G. Fontgalland, and S. Tedjini. A passive capacitive soil moisture and environment temperature UHF RFID based sensor for low cost agricultural applications. In 2017 SBMO/IEEE MTT$S$ International Microwave and Optoelectronics Conference (IMOC), pages 1-4, 2017. 


\section{Appendix A}

\section{Interrogator Code (Python)}

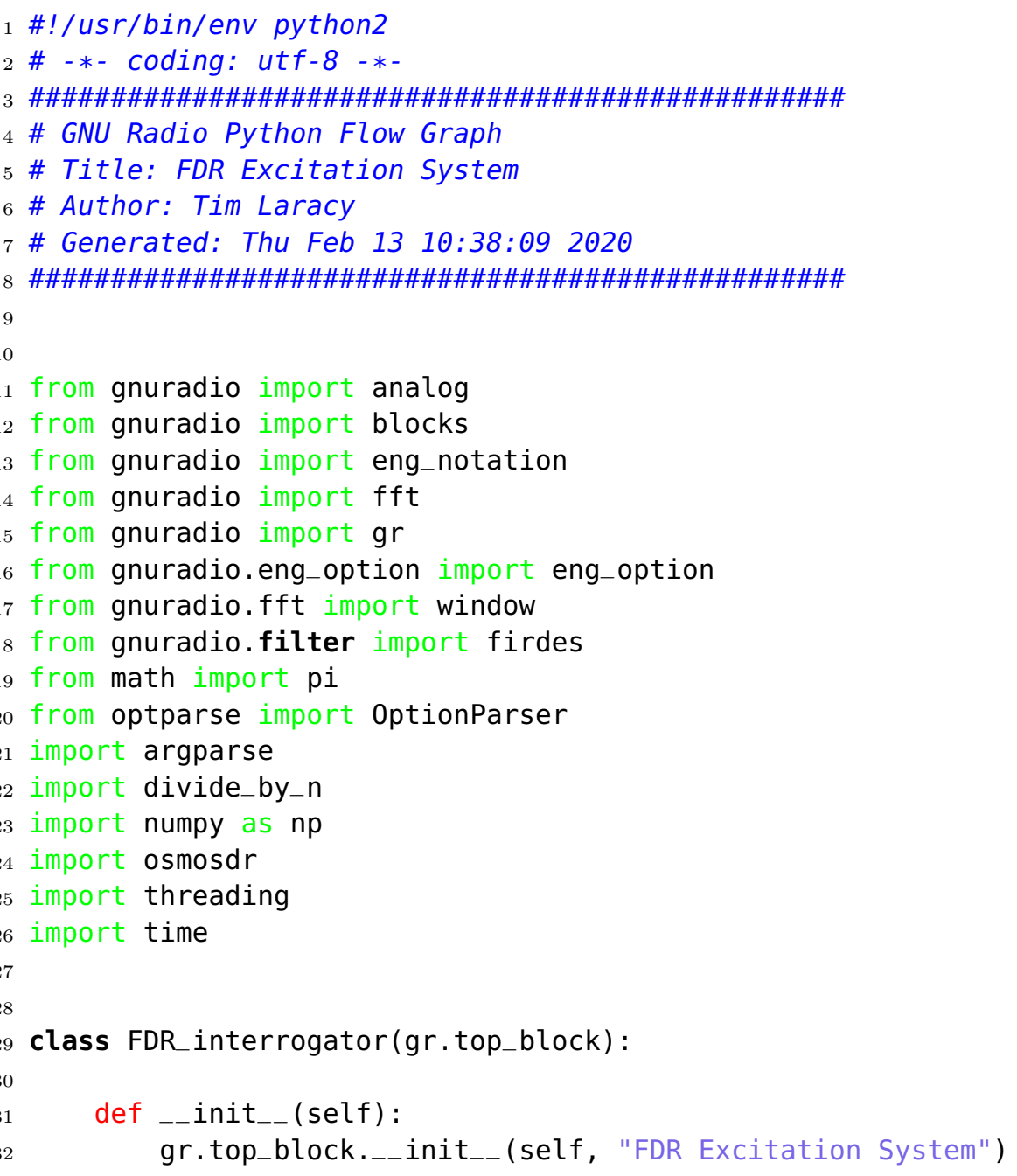




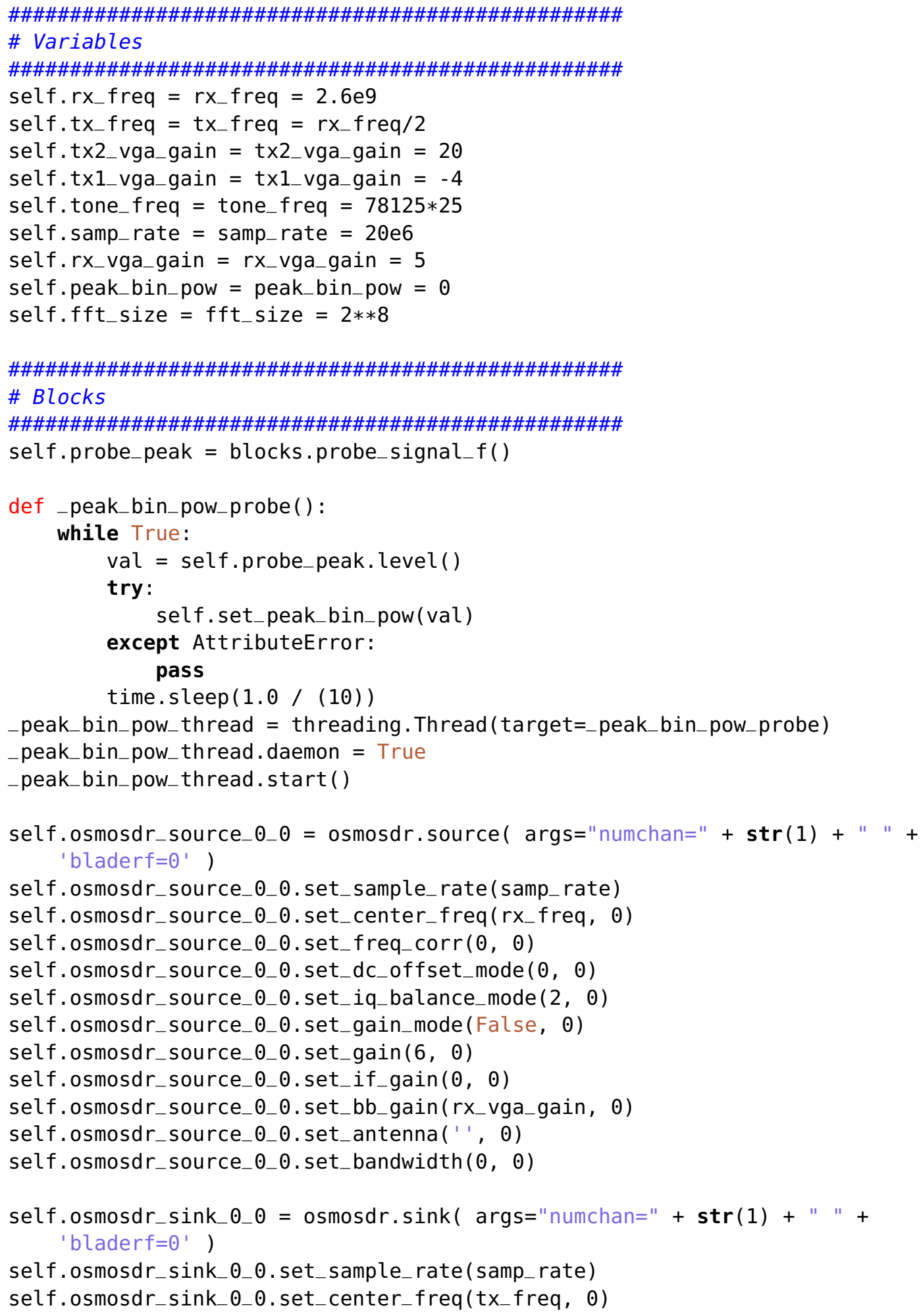




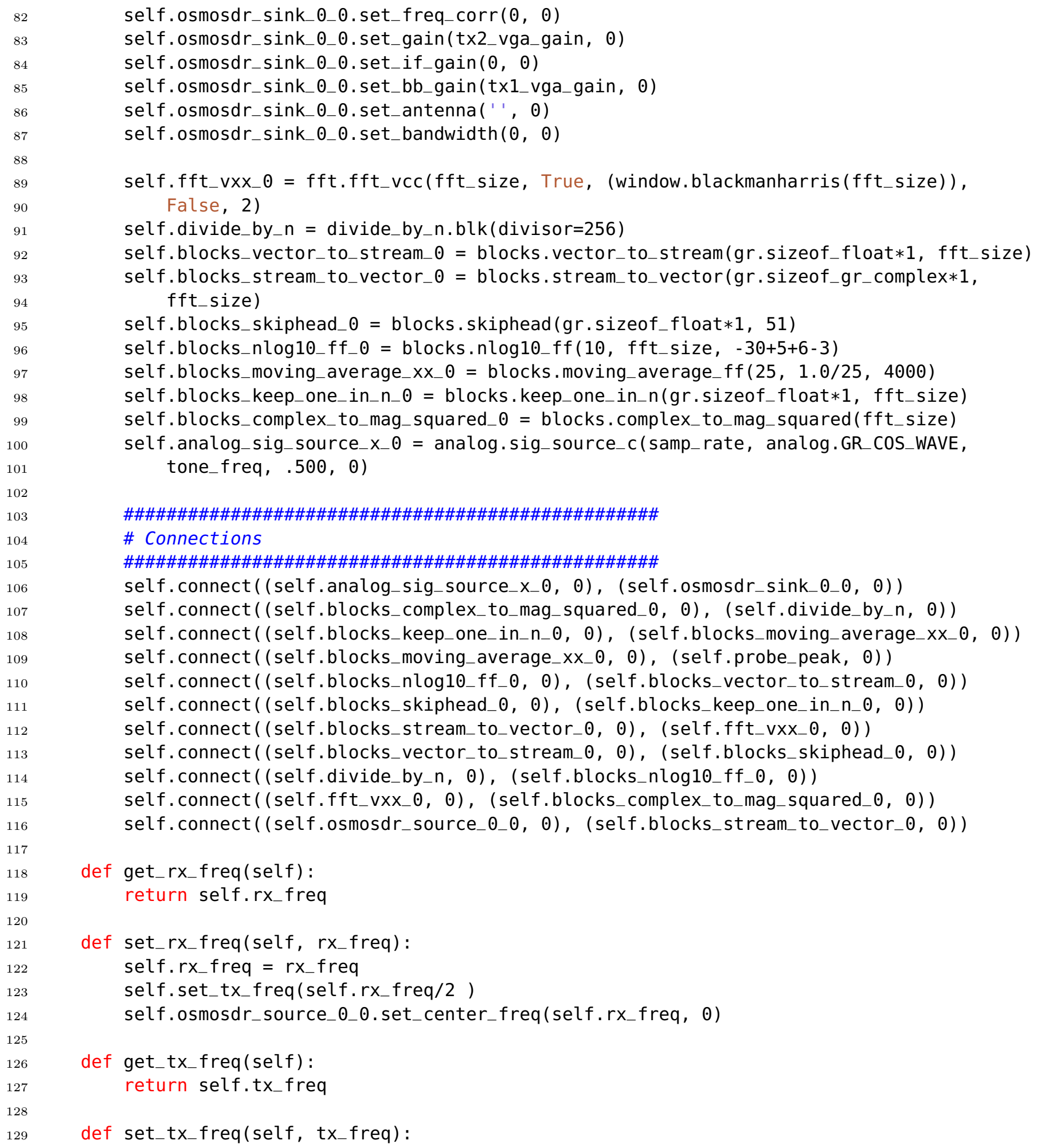

self.osmosdr_sink_o_ 0. set_freq_orr $(\theta, 0)$

self.osmosdr_sink_0_0.set_gain (tx2_vga_gain, $\theta$ )

self.osmosdr_sink_o_o.set_if_gain $(\theta, \theta)$

self.osmosdr_sink_0_0.set_bb_gain (tx1_vga_gain, $\theta$ )

self.osmosdr_sink_o_o.set_antenna( ' ', $\theta)$

self.osmosdr_sink_0_0.set_bandwidth $(\theta, 0)$

self.fft_vxx_0 = fft.fft_vcc(fft_size, True, (window.blackmanharris(fft_size)), False, 2)

self.divide_by_n = divide_by_n.blk(divisor=256)

self.blocks_vector_to_stream_0 = blocks.vector_to_stream(gr.sizeof_float $* 1$, fft_size)

self.blocks_stream_to_vector_ $\theta=$ blocks.stream_to_vector(gr.sizeof_gr_complex $* 1$, fft_size)

self.blocks_skiphead_ $\theta=$ blocks.skiphead(gr.sizeof_float $* 1,51)$

self.blocks_nlog10_ff_o = blocks.nlog10_ff(10, fft_size, $-30+5+6-3)$

self.blocks_moving_average_xx_0 = blocks.moving_average_ff $(25,1.0 / 25,4000)$

self.blocks_keep_one_in_n_o = blocks.keep_one_in_n(gr.sizeof_float $* 1$, fft_size) self.blocks_complex_to_mag_squared_ $\theta=$ blocks.complex_to_mag_squared (fft_size)

self.analog_sig_source_x_O = analog.sig_source_c(samp_rate, analog.GR_COS_WAVE, tone_freq, . .500, 0 )

\section{\#\#\#\#\#\#\#\#\#\#\#\#\#\#\#\#\#\#\#\#\#\#\#\#\#\#\#\#\#\#\#\#\#\#\#\#\#\#\#\#\#\#}

\# Connections

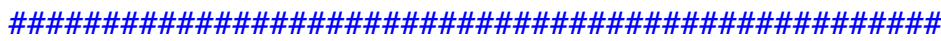

self.connect ( (self.analog_sig_source_x_o, $\theta)$, (self.osmosdr_sink_o_o, $\theta)$ )

self.connect ( (self.blocks_complex_to_mag_squared_ $\theta, 0)$, (self.divide_by_n, 0$)$ )

self.connect ( (self.blocks_keep_one_in_n_0, 0$)$, (self.blocks_moving_average_xx_0, 0$)$ )

self.connect ( (self.blocks_moving_average_xx_0, 0$)$, (self.probe_peak, 0$)$ )

self.connect ( (self.blocks_nlog10_ff_o, $\theta)$, (self.blocks_vector_to_stream_ $\theta, 0)$ )

self.connect ( (self.blocks_skiphead_ $\theta, \theta)$, (self.blocks_keep_one_in_n_ 0,0$)$ )

self.connect ( (self.blocks_stream_to_vector_ $\theta, 0)$, ( self.fft_vxx_ 0,0$)$ )

self.connect ( (self.blocks_vector_to_stream_0, 0$)$, (self.blocks_skiphead_0, 0$)$ )

self.connect ( (self.divide_by_n, 0$)$, (self.blocks_nlog10_ff_o, 0$)$ )

self.connect ( (self.fft_vxx_0, $\theta)$, (self.blocks_complex_to_mag_squared_ $\theta, \theta)$ )

self.connect ( (self.osmosdr_source_ $\left.\theta \_\theta, \theta\right)$, (self.blocks_stream_to_vector_ $\left.\theta, \theta\right)$ )

def get_rx_freq(self):

return self.rx_freq

def set_rx_freq(self, rx_freq):

self.rx_freq $=r x_{-} f r e q$

self.set_tx_freq(self.rx_freq/2)

self.osmosdr_source_o_o.set_center_freq(self.rx_freq, 0 )

def get_tx_freq(self):

return self.tx_freq

def set_tx_freq(self, tx_freq): 


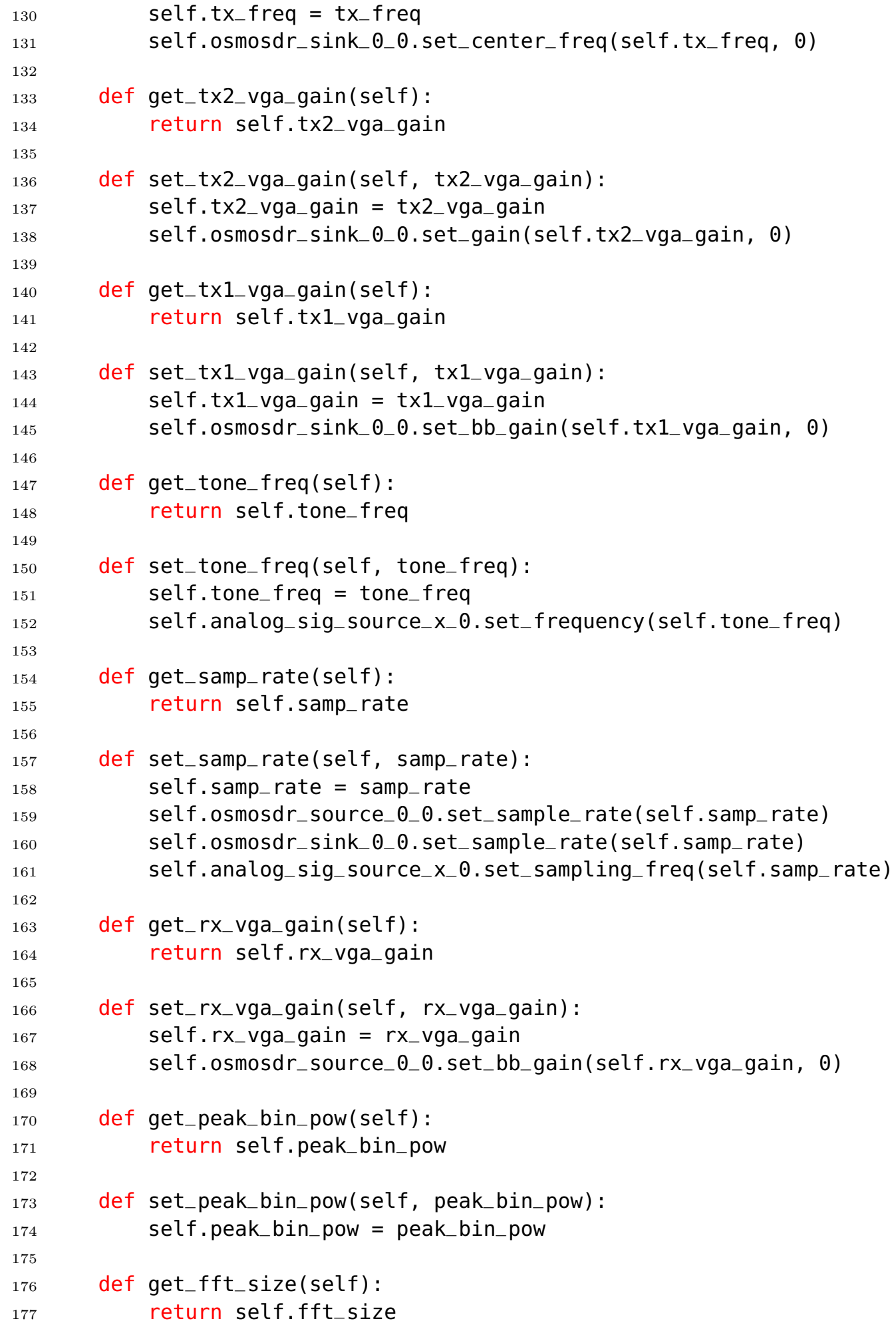




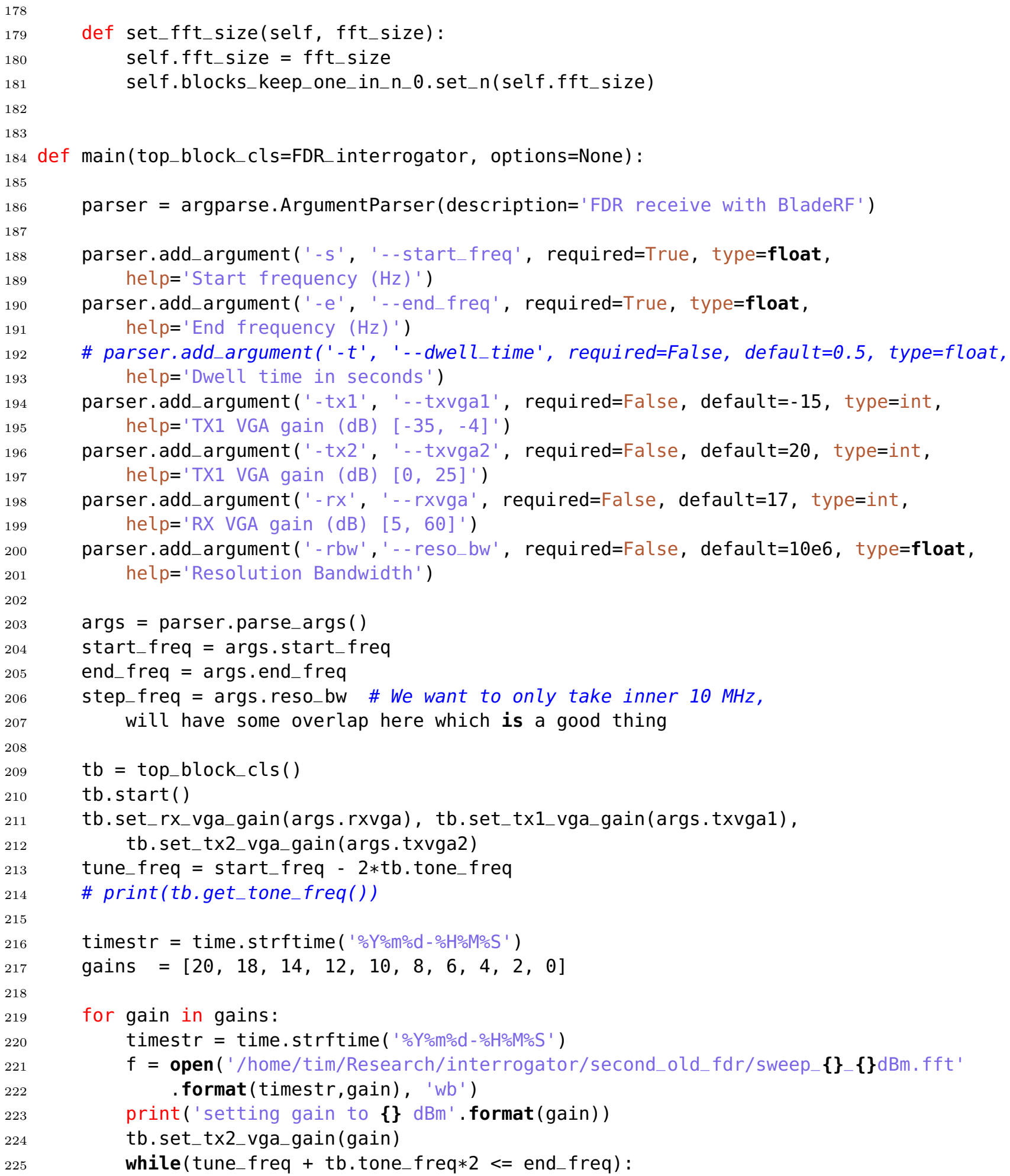




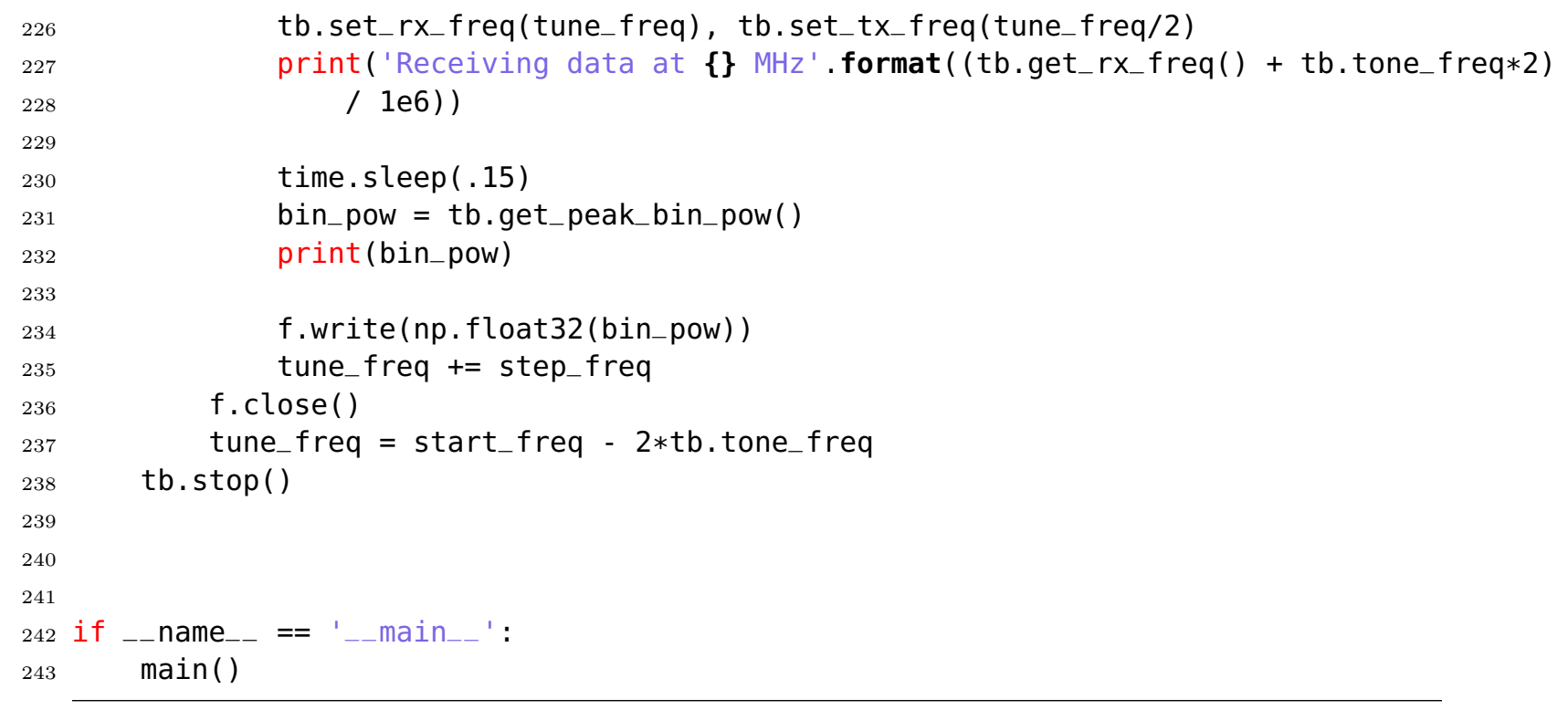

